\title{
Global stabilization of linear systems with bounds on the feedback and its successive derivatives
}

\author{
Jonathan Laporte, Antoine Chaillet and Yacine Chitour *
}

\begin{abstract}
We address the global stabilization of linear time-invariant (LTI) systems when the magnitude of the control input and its successive time derivatives, up to an order $p \in \mathbb{N}$, are bounded by prescribed values. We propose a static state feedback that solves this problem for any admissible LTI systems, namely for stabilizable systems whose internal dynamics has no eigenvalue with positive real part. This generalizes previous work done for single-input chains of integrators and rotating dynamics.
\end{abstract}

\section{Introduction}

The study of control systems subject to input constraints is motivated by the fact that signals delivered by physical actuators may be limited in amplitude, and may not evolve arbitrarily fast. An a priori bound on the amplitude of the control signal is usually referred to as input saturation whereas a bound on the variation of control signal is referred to as rate saturation (e.g [1]).

Stabilization of linear time-invariant systems (LTI for short) with input saturation has been widely studied in the literature. Such a system is given by

$$
\text { (S) } \dot{x}=A x+B u,
$$

where $x \in \mathbb{R}^{n}, u$ belongs to a bounded subset of $\mathbb{R}^{m}, A$ is an $n \times n$ matrix and $B$ is an $n \times m$ one. Global stabilization of $(S)$ can be achieved if and only if the LTI system is asymptotically null controllable with bounded controls, i.e., it can be stabilized in the absence of input constraint and the eigenvalues of $A$ have non positive real parts. Saturating a linear feedback law may fail at globally stabilizing $(S)$ as it was observed first in [2] and then [3] for the special case of integrator chains (i.e., when $A$ is the $n$-th Jordan block and $\left.B=\left(\begin{array}{lll}0 & \cdots & 0\end{array}\right)^{T}\right)$. As shown for instance in [4], optimal control can be used to define a globally stabilizing feedback for $(S)$ but, when the dimension is greater than 3, deriving a closed form for this stabilizer becomes extremely difficult. The first globally stabilizing feedback with rather simple closed form (nested saturations) was provided in [5] for chains of integrators and then in [6] for the general case. In [7], a global feedback stabilizer for $(S)$ was built by relying on control Lyapunov functions arising from a mere existence result. Other globally stabilizing feedback laws for $(S)$ have been proposed with an additional property of

\footnotetext{
*This research was partially supported by a public grant overseen by the French ANR as part of the Investissements d'Avenir program, through the iCODE institute, research project funded by the IDEX Paris-Saclay, ANR-11-IDEX-0003-02.

$\dagger$ J. Laporte, A. Chaillet and Y. Chitour are with L2S - Univ. Paris Sud - CentraleSupélec. 3, rue Joliot-Curie. 91192 - Gif sur Yvette, France. jonathan.laporte, antoine.chaillet, yacine.chitour@l2s.centralesupelec.fr
} 
robustness with respect to perturbations. In [8], using low-and-high gain techniques, a robust stabilizer was proposed to ensure semiglobal stability, meaning that the control gains can be tuned in such a way that the basin of attraction contains any prescribed compact subset of $\mathbb{R}^{n}$. This restriction has been removed in [9], where the authors provided a global feedback stabilizer for $(S)$ which is robust with respect to perturbations, based on an earlier idea due to Megretsky [10]. Nonetheless, the feedback laws of [9] and [10] require to solve a nonlinear optimization problem at every point $x \in \mathbb{R}^{n}$, which makes its practical implementation questionable. In [11], an easily implementable global feedback stabilizer for $(S)$ which is robust with respect to perturbations was proposed but it only covers the multiple integrator case and it is discontinuous since it is based on sliding mode techniques. Robust stabilization of $(S)$ was also addressed in [12] by relying on the control Lyapunov techniques developed in [7].

In contrast to stabilization of LTI systems subject to input saturation, there are much less results available in the literature regarding global stabilization under rate saturation, i.e., when the first time derivative of the control signal is also a priori bounded. In [13], the authors rely on a backstepping procedure to build a bounded globally stabilizing feedback with a bounded rate, but the methodology does not allow to $a$ priori impose a prescribed rate. In [14], a dynamic feedback law inspired from [10] is constructed and can even be generalized to take into account constraints on higher time derivatives of the control signal. However, as mentioned previously, the numerical efficiency of such feedbacks is definitely questionable. A rather involved global feedback stabilizer for $(S)$ achieving amplitude and rate saturations was also obtained in [15] for continuous time affine systems with a stable free dynamics. This corresponds in our setting to requiring that the matrix $A$ is stable, i.e., $A^{T}+A \leq 0$ (up to similarity). Finally, let us mention the references [16], [17] for semiglobal stabilization results and [18] for local stabilization results using LMIs and anti-windup design. One should also mention [19] where a nonlinear small gain theorem is given for the behaviour analysis of control systems with saturation.

The results presented here encompass input and rate saturations as special cases. More precisely, given an integer $p$, we construct a globally stabilizing feedback for $(S)$ such that the control signal and its $p$ first time derivatives, are bounded by arbitrary prescribed positive values, along all trajectories of the closed-loop system. This problem has already been solved by the authors in [20] for the multiple integrator and skewsymmetric cases. The solution given in that paper for the multiple integrator case consisted in considering appropriate nested saturation feedbacks. We also indicated in [20] that these feedbacks fail at ensuring global stability in the skew-symmetric case and we then provided an ad hoc feedback law for this specific case. Here, we solve the general case with a unified strategy.

The paper should be seen as a first theoretical step towards the global stabilization of an LTI system when the input signal is delivered by a dynamical actuator that limits the control action in terms of magnitude and $p$ first time derivatives. Further developments are needed to explicitly take into account the dynamics of such an actuator. Possible extensions of this work may also address the question of global stabilization by smooth feedback laws (i.e., $C^{\infty}$ with respect to time) when all successive derivatives need to be bounded by prescribed values.

The paper is organized as follows. In Section 2, we precisely state the problem we want to tackle, the needed definitions as well as the main results we obtain, namely Theorem 1 for the single input case and Theorem 2 for the multiple input case. Section 3 contains the proof of the main results. In section 3.1.1 we show that the proof of Theorem 1 is a consequence of two propositions. The first one (cf. Proposition 1), we show that the feedback proposed in Theorem 1 is indeed a globally stabilizing feedback for $(S)$. We actually prove a stronger result dealing with robustness properties of this feedback, as it is required in [5] and [6]. The second proposition (cf. Proposition 2) specifically deals with bounding the $p$ first derivatives of the control signal by relying on delicate estimates. Section 3.2.1 contains the proof of Theorem 2 which is a consequence of Proposition 1 and Proposition 3, the latter providing estimates on the successive time 
derivatives of the control signal. We close the paper by an Appendix, where we gather several technical results used throughout the paper.

Notations : We use $\mathbb{R}$ and $\mathbb{N}$ to denote the sets of real numbers and the set of non negative integers respectively. Given a set $I \subset \mathbb{R}$ and a constant $a \in \mathbb{R}$, we let $I_{\geq a}:=\{x \in I: x \geq a\}$. Given $m, k \in \mathbb{N}$, we define $\llbracket m, k \rrbracket:=\{l \in \mathbb{N}: l \in[m, k]\}$. For a given set $M$, the boundary of $M$ is denoted by $\partial M$. The factorial of $k$ is denoted by $k$ ! and the binomial coefficient is denoted $\left(\begin{array}{l}k \\ m\end{array}\right):=\frac{k !}{m !(k-m) !}$.

Given $k \in \mathbb{N}$ and $n, p \in \mathbb{N}_{\geq 1}$, we say that a function $f: \mathbb{R}^{n} \rightarrow \mathbb{R}^{p}$ is of class $C^{k}\left(\mathbb{R}^{n}, \mathbb{R}^{p}\right)$ if its differentials up to order $k$ exist and are continuous, and we use $f^{(k)}$ to denote the $k$-th order differential of $f$. By convention, $f^{(0)}:=f$.

Given $n, m \in \mathbb{N}_{\geq 1}, \mathbb{R}^{n, m}$ denotes the set of $n \times m$ matrices with real coefficients. The transpose of a matrix $A$ is denoted by $A^{T}$. The identity matrix of dimension $n$ is denoted by $\mathbb{I}_{n}$. We say that an eigenvalue of $A$ is critical if it has zero real part and we set $\mu(A):=s(A)+z(A)$ where $s(A)$ is the number of conjugate pairs of nonzero purely imaginary eigenvalues of $A$ (counting multiplicity), and $z(A)$ is the multiplicity of the zero eigenvalue of $A$. We define $A_{0}:=\left(\begin{array}{cc}0 & 1 \\ -1 & 0\end{array}\right)$, and $b_{0}:=\left(\begin{array}{l}0 \\ 1\end{array}\right)$.

We use $\|x\|$ to denote the Euclidean norm of an arbitrary vector $x \in \mathbb{R}^{n}$. Given $\delta>0$ and $f: \mathbb{R}_{\geq 0} \rightarrow \mathbb{R}^{n}$, we say that $f$ is eventually bounded by $\delta$, and we write $\|f(t)\| \leq_{e v} \delta$, if there exists $T>0$ such that $\|f(t)\| \leq \delta$ for all $t \geq T$.

\section{Problem statement and main results}

Given $n \in \mathbb{N}_{\geq 1}$ and $m \in \mathbb{N}_{\geq 1}$, consider the LTI system defined by

$$
\dot{x}=A x+B u,
$$

where $x \in \mathbb{R}^{n}, u \in \mathbb{R}^{m}, A \in \mathbb{R}^{n, n}$, and $B \in \mathbb{R}^{n, m}$. Assume that the pair $(A, B)$ is stabilizable and that all the eigenvalues of $A$ have non positive real parts. Recall that these assumptions on $(A, B)$ are necessary and sufficient for the existence of a bounded continuous state feedback $u=k(x)$ which globally asymptotically stabilizes the origin of (1): see [6].

Given an integer $p$ and a $(p+1)$-tuple of positive real numbers $\left(R_{j}\right)_{0 \leq j \leq p}$, we want to derive a feedback law whose magnitude and $p$-first time derivatives are bounded by $R_{j}, j \in \llbracket 0, p \rrbracket$.

Definition 1 (feedback law p-bounded by $\left(R_{j}\right)_{0 \leq j \leq p}$ ). Given $n \in \mathbb{N}_{\geq 1}, m \in \mathbb{N}_{\geq 1}$ and $p \in \mathbb{N}$, let $\left(R_{j}\right)_{0 \leq j \leq p}$ be $a(p+1)$-tuple of positive real numbers. We say that $v: \mathbb{R}^{n} \rightarrow \mathbb{R}^{m}$ is a feedback law $p$-bounded by $\left(R_{j}\right)_{0 \leq j \leq p}$ for system (11) if it is of class $C^{p}\left(\mathbb{R}^{n}, \mathbb{R}^{m}\right)$ and, for every trajectory of the closed-loop system $\dot{x}=A x+B v(x)$, the control signal $U: \mathbb{R}_{\geq 0} \rightarrow \mathbb{R}^{m}, t \mapsto U(t):=v(x(t))$ satisfies $\sup _{t \geq 0}\left\|U^{(j)}(t)\right\| \leq R_{j}$ for all $j \in \llbracket 0, p \rrbracket$. The function $v: \mathbb{R}^{n} \rightarrow \mathbb{R}^{m}$ is said to be a feedback law p-bounded for system (1), if there exist $(p+1)$-tuple of positive real numbers $\left(R_{j}\right)_{0 \leq j \leq p}$ such that $\mu(\cdot)$ is a feedback law $p$-bounded by $\left(R_{j}\right)_{0 \leq j \leq p}$ for system (1).

Based on this definition, we can write our stabilization problem of Bounded Higher Derivatives as follows.

Problem (BHD). Given $p \in \mathbb{N}$ and a $(p+1)$-tuple of positive real numbers $\left(R_{j}\right)_{0 \leq j \leq p}$, design a feedback law $v: \mathbb{R}^{n} \rightarrow \mathbb{R}^{m}$ such that the origin of the closed-loop system $\dot{x}=A x+B v(x)$ is globally asymptotically stable (GAS for short) and the feedback $v$ is a feedback law p-bounded by $\left(R_{j}\right)_{0 \leq j \leq p}$ for system (I). 
Our construction to solve Problem (BHD) will often use the property of Small Input Small State with linear gain $\left(\operatorname{SISS}_{L}\right.$ for short) developed in [6]. We recall below its definition

Definition $2\left(\operatorname{SISS}_{L},[6]\right)$. Given $\Delta>0$ and $N>0$, the control system $\dot{x}=f(x, u)$, with $x \in \mathbb{R}^{n}$ and $u \in \mathbb{R}^{m}$, is said to be $\operatorname{SISS}_{L}(\Delta, N)$ if, for all $\delta \in(0, \Delta]$ and all bounded measurable signal $e: \mathbb{R}_{\geq 0} \rightarrow \mathbb{R}^{m}$ eventually bounded by $\delta$, every solution of $\dot{x}=f(x, e)$ is eventually bounded by $N \delta$. A system is said to be $\operatorname{SISS}_{L}$ if it is $\operatorname{SISS}_{L}(\Delta, N)$ for some $\Delta, N>0$. An input-free system $\dot{x}=f(x)$ is called SISS $S_{L}$, if the control system $\dot{x}=f(x)+u$ is $\operatorname{SISS}_{L}$.

Remark 1. It follows readily from this definition that if $\dot{x}=f(x)$ is SISS $S_{L}$, then all solutions $\dot{x}=f(x)$ converge to the origin. Note, however, that the SISS $S_{L}$ property does not necessarily ensure GAS in the absence of input, as it does not imply stability of its origin.

When a feedback law ensures both global asymptotic stability and SISS $_{L}$, we refer to is an SISS $_{L^{-}}$ stabilizing feedback.

Definition 3 (SISS $L_{L}$-stabilizing feedback). Given a control system $\dot{x}=f(x, u)$ with $x \in \mathbb{R}^{n}$ and $u \in \mathbb{R}^{m}$, we say that a feedback law $v: \mathbb{R}^{n} \rightarrow \mathbb{R}^{m}$ is stabilizing if the origin of the closed-loop system $\dot{x}=f(x, v(x))$ is globally asymptotically stable. If, in addition, this closed-loop system is $S_{S I S S_{L}}$, then we say that $v$ is SISS $_{L}$-stabilizing.

As mentioned before the feedback law given in [20], which solves Problem (BHD) for the special case of multiple integrators, simply made use of nested saturations with carefully chosen saturation functions. We recall next why this feedback construction cannot work in general. For that purpose it is enough to consider the 2D simple oscillator case which is the control system given by $\dot{x}=\omega A_{0} x+b_{0} u$, with $x=\left(x_{1}, x_{2}\right)^{T}, u \in \mathbb{R}$ and $\omega>0$. This system is one of the two basic systems to be stabilized by means of a bounded feedback, as explained in [6]. One must then consider a stabilizing feedback law $u=-\sigma\left(k^{T} x\right)$, where $k=\left(k_{1}, k_{2}\right)^{T}$ is a fixed vector in $\mathbb{R}^{2}$ and $\sigma: \mathbb{R} \rightarrow \mathbb{R}$ is a saturation function, i.e., a bounded, continuously differentiable function satisfying $s \sigma(s)>0$ for $s \neq 0$ and $\sigma^{(1)}(0)>0$. Note that $k$ is chosen so that the linearized system at $(0,0)$ is Hurwitz. In particular it implies that $k_{2} \neq 0$. Pick now the following sequence of initial conditions $\left(l,-k_{1} l / k_{2}\right)_{l \geq 1}$. A straightforward computation yields that the first time derivative of the control along each trajectory satisfies $\dot{u}(0)=-\sigma^{(1)}(0) \omega l\left(k_{1}^{2} / k_{2}+k_{2}\right)$, which grows unbounded as $l$ tends to infinity. Therefore this feedback can not be a 1-bounded feedback.

In order to solve Problem (BHD) for the $2 D$ oscillator, we showed in [20] that a feedback law of the type $u_{k, \alpha}:=\frac{k^{T} x}{\left(1+\|x\|^{2}\right)^{\alpha}}$ with $k \in \mathbb{R}^{2}$ and $\alpha \geq 1 / 2$ does the job and it also solves Problem (BHD) in case the matrix $A$ in (1) is stable. However, we are not able to show whether $u_{k, \alpha}$ stabilizes or not the system in the case where $A:=\left(\begin{array}{cc}A_{0} & \mathbb{I}_{2} \\ 0 & A_{0}\end{array}\right)$. It turns out that the previous issue is as difficult as asking if a saturated linear feedback stabilizes or not the abovementioned 4D case, which is an open problem. It is therefore not immediate how to address the general case. This is why Theorem 1 is a non trivial extension of the solution of Problem (BHD) provided for the two-dimensional oscillator.

\subsection{Single input case}

For the case of single input systems the solution of Problem (PHB) is given by the following statement.

Theorem 1 (Single input). Given $n \in \mathbb{N}_{>0}$, consider a single input system $\dot{x}=A x+$ bu where $x \in \mathbb{R}^{n}, A \in \mathbb{R}^{n, n}$ and $b \in \mathbb{R}^{n, 1}$. Assume that $A$ has no eigenvalue with positive real part and that the pair $(A, b)$ is stabilizable. 
Then, given any $p \in \mathbb{N}$ and any $(p+1)$-tuple $\left(R_{j}\right)_{0 \leq j \leq p}$ of positive real numbers, there exist vectors $k_{i} \in \mathbb{R}^{n}$ and matrices $T_{i} \in \mathbb{R}^{n, n}, i \in \llbracket 1, \mu(A) \rrbracket$, such that the feedback law $v: \mathbb{R}^{n} \rightarrow \mathbb{R}$ defined as

$$
v(x)=-\sum_{j=1}^{\mu(A)} \frac{k_{j}^{T} x}{\left(1+\left\|T_{l} x\right\|^{2}\right)^{1 / 2}},
$$

is a feedback law p-bounded by $\left(R_{j}\right)_{0 \leq j \leq p}$ and SISS $_{L}$-stabilizing for system $\dot{x}=A x+b u$.

In view of Definition 3, the feedback law (2) globally asymptotically stabilizes the origin of (1), and thus solves Problem (BHD). We stress that, even though the exact computation of the control gains $k_{i}$ is quite involved (see proof in Section 3), the structure of the proposed feedback law (2) is rather simple. It should also be noted that, unlike the results developed in [20], this feedback law applies to any admissible single-input systems in a unified manner.

\subsection{Multiple input case}

To give the main result for LTI system with multiple input we need this following definition.

Definition 4 (Reduced controllability form). Given $n \in \mathbb{N}$ and $q \in \mathbb{N}$, a LTI system is said to be in reduced controllability form if it reads

$$
\begin{array}{rrr}
\dot{x}_{0}= & A_{00} x_{0}+A_{01} x_{1}+A_{02} x_{2}+\ldots+A_{0 q} x_{q}+ & b_{01} u_{1}+b_{02} u_{2}+\ldots+b_{0 q} u_{q}, \\
\dot{x}_{1}= & A_{11} x_{1}+A_{12} x_{2}+\ldots+A_{1 q} x_{q}+ & b_{11} u_{1}+b_{22} u_{2}+\ldots+b_{1 q} u_{q}, \\
\dot{x}_{2}= & A_{22} x_{2}+\ldots+A_{2 q} x_{q}+ & b_{22} u_{2}+\ldots+b_{2 q} u_{q}, \\
\vdots & & \\
\dot{x}_{q}= & A_{q q} x_{q}+ & \\
& & b_{q q} u_{q},
\end{array}
$$

where, for some $(q+1)$-tuple $\left(n_{i}\right)_{0 \leq i \leq q+1}$ in $\mathbb{N} \times\left(\mathbb{N}_{>0}\right)^{q}$ with $\sum_{i=0}^{q} n_{i}=n, A_{00} \in \mathbb{R}^{n_{0}, n_{0}}$ is Hurwitz, for every $i \in \llbracket 1, q \rrbracket$ all the eigenvalues of $A_{i i} \in \mathbb{R}^{n_{i}, n_{i}}$ are critical, $b_{i i} \in \mathbb{R}^{n_{i}, 1}$ and the pairs $\left(A_{i i}, b_{i i}\right)$ are controllable.

From Lemma 5.1 in [6], it is then clear that without loss of generality, in our case, we can consider that system (1) is already given in the reduced controllability form. We can now establish the solution of Problem (BHD) for the multiple input case.

Theorem 2 (Multiple input). Let $p \in \mathbb{N}$ and $(p+1)$-tuple $\left(R_{j}\right)_{0 \leq j \leq p}$ of positive real numbers. Given $n \in \mathbb{N}$ and $q \in \mathbb{N}$, consider system (3). Then, there exist q feedback laws $\kappa_{1}, \ldots, \kappa_{q}$ such that:

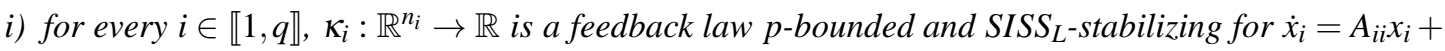
$b_{i i} u_{i}$;

ii) the feedback law $\mu=\left[\mu_{1}, \ldots, \mu_{q}\right]^{T}$ given by

$$
\begin{aligned}
\mu_{i}\left(x_{i}, \ldots, x_{q}\right) & :=\frac{\kappa_{i}\left(x_{i}\right)}{\left(1+\left\|x_{i+1}\right\|^{2}+\ldots+\left\|x_{q}\right\|^{2}\right)^{p+1}}, \quad \forall i \in \llbracket 1, q-1 \rrbracket, \\
\mu_{q}\left(x_{q}\right) & :=\kappa_{q}\left(x_{q}\right),
\end{aligned}
$$

is a feedback law p-bounded by $\left(R_{j}\right)_{0 \leq j \leq p}$ and $S I S S_{L^{-}}$-stabilizing for system (3).

This statement provides a unified control law solving Problem (BHD) for all admissible LTI systems. It allows in particular multi-input systems, which was not covered in [20]. 


\section{Proof of the main results}

\subsection{Proof of Theorem 1}

In this section, we prove Theorem 1. For that purpose, we first reduce the argument to establishing of Propositions 1 and 2 given below. The first one indicates that the feedback given in Theorem 1 is $\operatorname{SISS}_{L}$ stabilizing for $(S)$ in the case of single input. The second proposition provides an estimate of the successive time derivatives of the control signal.

\subsubsection{Reduction of the proof of Theorem 1 to the proofs of Propositions 1 and 2}

Let $n \in \mathbb{N}_{\geq 1}, p \in \mathbb{N}$ and $\left(R_{j}\right)_{0 \leq j \leq p}$ be a $(p+1)$-tuple of positive real numbers. Define $\underline{R}:=\min _{j \in \llbracket 0, p \rrbracket} R_{j}$. Consider a single input linear system $\dot{x}=A x+b u$ where $x \in \mathbb{R}^{n}, A$ and $b$ are $n \times n$ and $n \times 1$ matrices respectively. We assume that the pair $(A, b)$ is stabilizable and that all the eigenvalues of $A$ have non positive real parts. As observed in [6], it is sufficient to consider the case where the pair $(A, b)$ is controllable and all eigenvalues of $A$ are critical. Indeed, since $(A, b)$ is stabilizable there exists a linear change of coordinates transforming $A$ and $b$ into $\left(\begin{array}{ll}A_{1} & 0 \\ 0 & A_{2}\end{array}\right)$ and $\left(\begin{array}{l}b_{1} \\ b_{2}\end{array}\right)$, where $A_{1}$ is Hurwitz, the eigenvalues of $A_{2}$ are critical and the pair $\left(A_{2}, b_{2}\right)$ is controllable. Then, it is immediate to see that we only have to treat the case where $A$ has only critical eigenvalues. From now on, we therefore assume that $A$ has only eigenvalues with zero real parts, and that the pair $(A, b)$ is controllable.

Our construction uses the following linear change of coordinates given by [6, Lemma 5.2]. This decomposition puts the original system in a triangular form made of one-dimensional integrators and twodimensional oscillators.

Lemma 1 (Lemma 5.2 in [6]). Let $\dot{x}=A x+b u, x \in \mathbb{R}^{n}, u \in \mathbb{R}$, be a controllable single input linear system. Assume that all the eigenvalues of A are critical. Let $\pm i \omega_{1}, \ldots, \pm i \omega_{s(A)}$ be the nonzero eigenvalues of $A$. Let $\left(a_{2}, \ldots, a_{\mu(A)}\right)$ be a family of positive numbers. Define

$$
\begin{aligned}
\theta_{i, k} & =1, \quad \text { for } \quad k=i+1 \\
\theta_{i, k} & =\prod_{h=i}^{k-2} 1 / a_{h+1}, \quad \text { for } \quad i+2 \leq k \leq \mu(A)+1 .
\end{aligned}
$$

Then there is a linear change of coordinates that puts $\dot{x}=A x+b u$ in the form

$$
\begin{aligned}
& \dot{y}_{i}=\omega_{i} A_{0} y_{i}+b_{0} \sum_{k=i+1}^{s(A)} \theta_{i, k} b_{0}^{T} y_{k}+b_{0} \sum_{k=s(A)+1}^{\mu(A)} \theta_{i, k} y_{k}+\theta_{i, \mu(A)+1} b_{0} u, \quad i=1, \ldots, s(A), \\
& \dot{y}_{i}=\sum_{k=i+1}^{\mu(A)} \theta_{i, k} y_{k}+\theta_{i, \mu(A)+1} u, \quad i=s(A)+1, \ldots, \mu(A)-1, \\
& \dot{y}_{\mu(A)}=u, \\
& \text { where } y_{i} \in \mathbb{R}^{2} \text { for } i=1, \ldots, s(A) \text {, and } y_{i} \in \mathbb{R} \text { for } i=s(A)+1, \ldots, \mu(A) \text {. }
\end{aligned}
$$

With no loss of generality, we prove Theorem 1 for system $\left(7\right.$, where the positive constants $\left(a_{2}, \ldots, a_{\mu(A)}\right)$ 
will be fixed later. Let $a_{1}$ be a positive constant. We rely on a candidate feedback $v: \mathbb{R}^{n} \rightarrow \mathbb{R}$ under the form

$$
\kappa(y)=-\sum_{i=1}^{s(A)} \frac{Q_{i, \mu(A)} b_{0}^{T} y_{i}}{\left(1+\sum_{m=i}^{\mu(A)}\left\|y_{m}\right\|^{2}\right)^{1 / 2}}-\sum_{i=s(A)+1}^{\mu(A)} \frac{Q_{i, \mu(A)} y_{i}}{\left(1+\sum_{m=i}^{\mu(A)}\left\|y_{m}\right\|^{2}\right)^{1 / 2}},
$$

with

$$
Q_{i, \mu(A)}:=\prod_{l=i}^{\mu(A)} a_{l} .
$$

It therefore remains to choose the positive constants $a_{1}, \ldots, a_{\mu(A)}$ such that the feedback law (8) is a feedback law $p$-bounded by $\left(R_{j}\right)_{0 \leq j \leq p}$, and $S I S S_{L}$-stabilizing for system (7). For that aim, we rely on the next two propositions, respectively proven in Sections 3.1.2 and 3.1.3.

Proposition 1. Let $\dot{x}=A x+b u, x \in \mathbb{R}^{n}, u \in \mathbb{R}$, be a controllable single input linear system. Assume that all the eigenvalues of $A$ are critical. Let $\pm i \omega_{1}, \ldots, \pm i \omega_{s(A)}$ be the nonzero eigenvalues of $A$. Then, there exist $\mu(A)-1$ functions $\bar{a}_{i}: \mathbb{R}_{>0} \rightarrow \mathbb{R}_{>0}, i \in \llbracket 1, \mu(A)-1 \rrbracket$ such that for any constants $a_{1}, \ldots, a_{\mu(A)}$ satisfying

$$
a_{\mu(A)} \in(0,1], \quad a_{i} \in\left(0, \bar{a}_{i}\left(a_{i+1}\right)\right], \quad \forall i \in \llbracket 1, \mu(A)-1 \rrbracket,
$$

the feedback law (8) is SISS $_{L}$-stabilizing for system (7).

Proposition 2. Let $\dot{x}=A x+b u, x \in \mathbb{R}^{n}, u \in \mathbb{R}$, be a controllable single input linear system. Assume that all the eigenvalues of $A$ are critical. Let $\pm i \omega_{1}, \ldots, \pm i \omega_{s(A)}$ be the nonzero eigenvalues of $A$. Let $a_{i}, i \in$ $\llbracket 1, \mu(A) \rrbracket$, be positive constants in $(0,1]$. Then, there exist a positive constant $c_{\mu}$, and continuous functions $c_{i}: \mathbb{R}_{>0}^{\mu(A)-i} \rightarrow \mathbb{R}_{>0}, i \in \llbracket 1, \mu(A)-1 \rrbracket$, such that for any trajectory of the closed-loop system (7) with the feedback law (8), the control signal $U: \mathbb{R}_{\geq 0} \rightarrow \mathbb{R}$ defined by $U(t):=v(y(t))$ for all $t \geq 0$ satisfies, for all $k \in \llbracket 0, p \rrbracket$,

$$
\left|U^{(k)}(t)\right| \leq a_{\mu} c_{\mu(A)}+\sum_{i=1}^{\mu(A)-1} a_{i} c_{i}\left(a_{\mu(A)}, \ldots, a_{i+1}\right), \quad \forall t \geq 0 .
$$

Pick $a_{\mu(A)} \in(0,1]$ in such a way that

$$
a_{\mu(A)} \leq \frac{\underline{R}}{(p+1) c_{\mu(A)}} .
$$

Choose recursively $a_{i} \in(0,1], i=\mu(A)-1, \ldots, 1$, such that

$$
a_{i} \leq \bar{a}_{i}\left(a_{i+1}\right), \quad a_{i} \leq \frac{\underline{R}}{(p+1) c_{i}\left(a_{\mu(A)}, \ldots, a_{i+1}\right)},
$$

where the functions $c_{i}$ appearing above are defined in Proposition 2 By Proposition 1, the feedback law (8) is $S I S S_{L}$-stabilizing for system (7). Moreover, as a consequence of Proposition 2 for any trajectory of the closed-loop system (7) with the feedback law (8), the control signal $U: \mathbb{R}_{\geq 0} \rightarrow \mathbb{R}$ defined by $U(t):=v(y(t))$ for all $t \geq 0$ satisfies $\sup _{t \geq 0}\left|U^{(k)}(t)\right| \leq \underline{R}$ for all $k \in \llbracket 0, p \rrbracket$. Thus, the feedback law (8) is a feedback law $p$-bounded by $\left(R_{j}\right)_{0 \leq j \leq p}$ for system (7). Since there is a linear change of coordinate $(y=T x)$ that puts (7) into the original form $\dot{x}=A x+b u$, the feedback law defined given in (2) can be picked as

$$
v(x):=\kappa(T x)
$$

and it is a feedback law $p$-bounded by $\left(R_{j}\right)_{0 \leq j \leq p}$, and $S I S S L_{L}$-stabilizing for (1). To sum up, the proof of Theorem 1 boils down to establishing Propositions 1 and 2 


\subsubsection{Proof of Proposition 1}

Proposition 1 is proved by induction on $\mu(A)$. More precisely, we show that the following property holds true for every positive integer $\mu$.

$\left(P_{\mu}\right)$ : Given any $\mu \in \mathbb{N}_{\geq 1}$, let $s, z \in \mathbb{N}$ be such that $s+z=\mu$ and $\omega_{1}, \ldots, \omega_{s}$ be positive constants. Then there exist $\mu-1$ functions $\bar{a}_{i}: \mathbb{R}_{>0} \rightarrow \mathbb{R}_{>0}, i \in \llbracket 1, \mu-1 \rrbracket$ such that for any constants $a_{1}, \ldots, a_{\mu}$ satisfying

$$
a_{\mu} \in(0,1], \quad a_{i} \in\left(0, \bar{a}_{i}\left(a_{i+1}\right)\right], \quad \forall i \in \llbracket 1, \mu-1 \rrbracket,
$$

the feedback law (8) is $\operatorname{SISS}_{L}$-stabilizing for system (7), with $\mu(A)=\mu, s(A)=s$, and $z(A)=z$. Moreover the linearization of this closed-loop system around the origin is asymptotically stable.

In order to start the argument, we give intermediate results whose proofs are given in Appendix and which will be used for the initialization step of the induction and the inductive step. The first statement establishes $S I S S_{L}$ for the one-dimensional integrator.

Lemma 2. Let $\varepsilon>1$. For every $\beta>0$, the scalar system given by

$$
\dot{x}=-\beta \frac{x}{\left(1+x^{2}\right)^{1 / 2}}
$$

is $\operatorname{SISS}_{L}\left(\frac{\beta}{2}, \frac{2 \varepsilon}{\beta}\right)$, its origin is GAS and its linearisation around zero is $A S$.

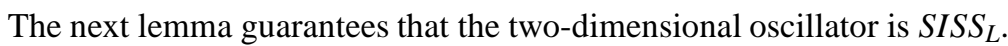

Lemma 3. For every $\omega>0$, there exist $\Gamma, N>0$ such that for any $\beta \in(0,1]$ the two-dimensional system given by

$$
\dot{x}=\omega A_{0} x-\beta b_{0} \frac{b_{0}^{T} x}{\left(1+\|x\|^{2}\right)^{1 / 2}}
$$

is $\operatorname{SISS}_{L}\left(\beta \Gamma, \frac{N}{\beta}\right)$, its origin is GAS and its linearisation around zero is $A S$.

We now start the inductive proof of $\left(P_{\mu}\right)$. For $\mu=1$, we have to consider two cases. Either $z=1$ and $s=0$ corresponding to the simple integrator

$$
\dot{y}_{1}=u, \quad \text { with } \quad u=\kappa\left(y_{1}\right)=-a_{1} \frac{y_{1}}{\left(1+y_{1}^{2}\right)^{1 / 2}},
$$

or $s=1$ and $z=0$ corresponding to the simple oscillator

$$
\dot{y}_{1}=\omega_{1} A_{0} y_{1}+b_{0} u, \quad \text { with } \quad u=\kappa\left(y_{1}\right)=-a_{1} \frac{b_{0}^{T} y_{1}}{\left(1+\left\|y_{1}\right\|^{2}\right)^{1 / 2}},
$$

for some $\omega_{1}>0$. In both cases, $\left(P_{1}\right)$ can be readily deduced by invoking Lemma 2 and 3 respectively. Given $\mu \in \mathbb{N}_{>0}$, assume that $\left(P_{\mu}\right)$ holds. In order to establish $\left(P_{\mu+1}\right)$, it is sufficient to consider the following two cases:

case i) $z=\mu+1$, i.e, all the eigenvalues of $A$ are zero (multiple integrator);

case ii) $s \geq 1$, i.e some eigenvalues of $A$ have non zero imaginary part (multiple integrator with rotating modes).

In both cases we reduce our problem to the choice of only one constant $a_{1}$ using the inductive hypothesis. 
case i) Let $\left(a_{1}, \ldots, a_{\mu+1}\right)$ be a set of positive numbers to be chosen later. Consider the multiple integrator given by

$$
\begin{aligned}
\dot{y}_{i} & =\sum_{k=i+1}^{\mu+1} \theta_{i, k} y_{k}+\theta_{i, \mu+2} u, \quad i=1, \ldots, \mu, \\
\dot{y}_{\mu+1} & =u,
\end{aligned}
$$

where $y_{i} \in \mathbb{R}$ for $i=1, \ldots, \mu+1$. Let $\tilde{y}=\left[y_{2}, \ldots, y_{\mu+1}\right]^{T}$. We then can rewrite this system as

$$
\begin{aligned}
\dot{y}_{1} & =\sum_{k=2}^{\mu+1} \theta_{i, k} y_{k}+\theta_{i, \mu+2} u, \\
\dot{\tilde{y}} & =\tilde{A} \tilde{y}+\tilde{b} u,
\end{aligned}
$$

for some matrices $\tilde{A}$ and $\tilde{b}$ of appropriate dimensions. From the inductive hypothesis, there exist $\mu-1$ functions $\bar{a}_{i}: \mathbb{R}_{>0} \rightarrow \mathbb{R}_{>0}$ for $i \in \llbracket 2, \mu \rrbracket$ such that for any set of positive constants $a_{2}, \ldots, a_{\mu+1}$ satisfying $a_{2}, \ldots, a_{\mu+1}$ satisfying $a_{\mu+1} \in(0,1]$ and $0<a_{i} \leq \bar{a}_{i}\left(a_{i+1}\right)$, for each $i \in \llbracket 2, \mu \rrbracket$, the feedback law $\tilde{\kappa}: \mathbb{R}^{\mu} \rightarrow \mathbb{R}$ defined by

$$
\tilde{\kappa}(\tilde{y})=-\sum_{i=2}^{\mu+1} \frac{Q_{i, \mu+1} y_{i}}{\left(1+\sum_{m=i}^{\mu+1}\left\|y_{m}\right\|^{2}\right)^{1 / 2}}
$$

is $\operatorname{SISS}_{L}$-stabilizing for $\dot{\tilde{y}}=\tilde{A} \tilde{y}+\tilde{b} u$. Choose $\left(a_{2}, \ldots, a_{\mu+1}\right)$ satisfying the above conditions. The feedback law (8) is then given by

$$
\kappa(y)=-\tilde{\kappa}(\tilde{y})-a_{1} Q_{2, \mu+1} \frac{y_{1}}{\left(1+\sum_{m=1}^{\mu+1}\left\|y_{m}\right\|^{2}\right)^{1 / 2}} .
$$

Since $\theta_{1, \mu+2} Q_{k, \mu+1}=\theta_{1, k}$ for all $k \in \llbracket 2, \mu+1 \rrbracket($ see (6) and (9)), the closed-loop system can be rewritten as

$$
\begin{aligned}
\dot{y}_{1} & =-a_{1} \frac{y_{1}}{\left(1+\left\|y_{1}\right\|^{2}\right)^{1 / 2}}+a_{1} \rho_{1}(y)+g_{1}(\tilde{y}), \\
\dot{y} & =\tilde{A} \tilde{y}-\tilde{b} \tilde{\kappa}(\tilde{y})-\tilde{b} a_{1} f_{1}(y),
\end{aligned}
$$

with

$$
\begin{aligned}
& \rho_{1}(y)=\frac{y_{1}}{\left(1+\left\|y_{1}\right\|^{2}\right)^{1 / 2}}\left(1-\frac{\left(1+\left\|y_{1}\right\|^{2}\right)^{1 / 2}}{\left(1+\sum_{m=1}^{\mu+1}\left\|y_{m}\right\|^{2}\right)^{1 / 2}}\right), \\
& g_{1}(\tilde{y})=\sum_{k=2}^{\mu+1} \theta_{1, k} y_{k}\left(1-\frac{1}{\left(1+\sum_{m=k}^{\mu+1}\left\|y_{m}\right\|^{2}\right)^{1 / 2}}\right), \\
& f_{1}(y)=\frac{Q_{2, \mu+1} y_{1}}{\left(1+\sum_{m=1}^{\mu+1}\left\|y_{m}\right\|^{2}\right)^{1 / 2}} .
\end{aligned}
$$

We now move to the other case where the dynamics involves multiple integrators with rotating modes. 
case ii) Let $\left(a_{1}, \ldots, a_{\mu+1}\right)$ be a set of positive constants to be chosen later. Let $s \in \mathbb{N}_{\geq 1}$, and $z \in \mathbb{N}$ be such that $\mu=s+z$. Let $\omega_{1}, \ldots, \omega_{s}$ be a set of non zero real numbers. Consider the following linear control system

$$
\begin{aligned}
\dot{y}_{i} & =\omega_{i} A_{0} y_{i}+b_{0} \sum_{k=i+1}^{s} \theta_{i, k} b_{0}^{T} y_{k}+b_{0} \sum_{k=s+1}^{\mu+1} \theta_{i, k} y_{k}+\theta_{i, \mu+2} b_{0} u, \quad i=1, \ldots, s, \\
\dot{y}_{i} & =\sum_{k=i+1}^{\mu+1} \theta_{i, k} y_{k}+\theta_{i, \mu+2} u, \quad i=s+1, \ldots, \mu, \\
\dot{y}_{\mu+1} & =u
\end{aligned}
$$

where $y_{i} \in \mathbb{R}^{2}$ for $i=1, \ldots, s$, and $y_{i} \in \mathbb{R}$ for $i=s+1, \ldots, \mu+1$. Let $\tilde{y}=\left[y_{2}, \ldots, y_{\mu+1}\right]^{T}$. We then can rewrite this system as follows

$$
\begin{aligned}
& \dot{y}_{1}=\omega_{1} A_{0} y_{1}+b_{0} \sum_{k=i+1}^{s} \theta_{i, k} b_{0}^{T} y_{k}+b_{0} \sum_{k=s+1}^{\mu+1} \theta_{i, k} y_{k}+\theta_{i, \mu+2} b_{0} u \\
& \dot{\tilde{y}}=\tilde{A} \tilde{y}+\tilde{b} u .
\end{aligned}
$$

From the inductive hypothesis, there exist $\mu-1$ functions $\bar{a}_{i}: \mathbb{R}_{>0} \rightarrow \mathbb{R}_{>0}$ for $i \in \llbracket 2, \mu \rrbracket$ such that for any set of positive constant $a_{2}, \ldots, a_{\mu+1}$ satisfying $a_{\mu+1} \in(0,1]$ and $0<a_{i} \leq \bar{a}_{i}\left(a_{i+1}\right)$, for each $i \in \llbracket 2, \mu \rrbracket$, the feedback law $\tilde{\kappa}: \mathbb{R}^{\mu} \rightarrow \mathbb{R}$ defined by

$$
\tilde{\kappa}(\tilde{y})=-\sum_{i=2}^{s} \frac{Q_{i, \mu+1} b_{0}^{T} y_{i}}{\left(1+\sum_{m=i}^{\mu+1}\left\|y_{m}\right\|^{2}\right)^{1 / 2}}-\sum_{i=s+1}^{\mu+1} \frac{Q_{i, \mu+1} y_{i}}{\left(1+\sum_{m=i}^{\mu+1}\left\|y_{m}\right\|^{2}\right)^{1 / 2}}
$$

is $S I S S_{L}$-stabilizing for $\dot{\tilde{y}}=\tilde{A} \tilde{y}+\tilde{b} u$. Choose $a_{2}, \ldots, a_{\mu+1}$ satisfying the above conditions. The feedback law (8) is then given by

$$
\kappa(y)=-\tilde{\kappa}(\tilde{y})-a_{1} Q_{2, \mu+1} \frac{b_{0}^{T} y_{1}}{\left(1+\sum_{m=1}^{\mu+1}\left\|y_{m}\right\|^{2}\right)^{1 / 2}} .
$$

By noticing that $\theta_{1, \mu+2} Q_{k, \mu+1}=\theta_{1, k}$ for all $k \in \llbracket 2, \mu+1 \rrbracket$ (see (6) and (9)), the closed-loop system can be rewritten as

$$
\begin{aligned}
\dot{y}_{1} & =\omega_{1} A_{0} y_{1}-a_{1} b_{0} \frac{b_{0}^{T} y_{1}}{\left(1+\left\|y_{1}\right\|^{2}\right)^{1 / 2}}+a_{1} b_{0} \rho_{1}(y)+b_{0} g_{1}(\tilde{y}), \\
\dot{\tilde{y}} & =\tilde{A} \tilde{y}-\tilde{b} \tilde{\kappa}(\tilde{y})-\tilde{b} a_{1} f_{1}(y),
\end{aligned}
$$


with

$$
\begin{aligned}
\rho_{1}(y) & =\frac{b_{0}^{T} y_{1}}{\left(1+\left\|y_{1}\right\|^{2}\right)^{1 / 2}}\left(1-\frac{\left(1+\left\|y_{1}\right\|^{2}\right)^{1 / 2}}{\left(1+\sum_{m=1}^{\mu+1}\left\|y_{m}\right\|^{2}\right)^{1 / 2}}\right), \\
g_{1}(\tilde{y})= & \sum_{k=2}^{s} \theta_{1, k} b_{0}^{T} y_{k}\left(1-\frac{1}{\left(1+\sum_{m=k}^{\mu+1}\left\|y_{m}\right\|^{2}\right)^{1 / 2}}\right)+\sum_{k=s+1}^{\mu+1} \theta_{1, k} y_{k}\left(1-\frac{1}{\left(1+\sum_{m=k}^{\mu+1}\left\|y_{m}\right\|^{2}\right)^{1 / 2}}\right), \\
f_{1}(y)= & \frac{Q_{2, \mu+1} b_{0}^{T} y_{1}}{\left(1+\sum_{m=1}^{\mu+1}\left\|y_{m}\right\|^{2}\right)^{1 / 2}} .
\end{aligned}
$$

In both cases, it remains to show that there exists a function $\bar{a}_{1}$ such that if $a_{1} \in\left(0, \overline{a_{1}}\right]$ then the closed-loop systems (14) and (19) are $S I S S_{L}$, globally asymptotically stable with respect to the origin, and theirs respective linearizations at zero are asymptotically stable. It is sufficient to prove that the closed-loop systems are $S I S S_{L}$ and their linearization at zero are asymptotically stable. Indeed, from Remark1 the $S I S S_{L}$ property guarantees the convergence of any solution of the closed-loop with no input. If moreover the linearized system is asymptotically stable, then the globally asymptotic stability of zero follows readily.

For any $a_{1}>0$, the linearization at zero of the $y_{1}$-subsystem in (14) (respectively (19)) is asymptotically stable since it is given by $\dot{y}_{1}=-a_{1} y_{1}$ (respectively $\left.\dot{y}_{1}=\left(\omega_{1} A_{0}-a_{1} b_{0} b_{0}^{T}\right) y_{1}\right)$. Moreover, the linearization at zero of the $\tilde{y}$-subsystem in (14) (respectively (19)) is given by $\dot{\tilde{y}}=\left(\tilde{A}-\tilde{b} \tilde{\kappa}^{(1)}(0)\right) \tilde{y}-a_{1} \tilde{b} y_{1}$ (respectively $\left.\dot{\tilde{y}}=\left(\tilde{A}-\tilde{b} \tilde{\kappa}^{(1)}(0)\right) \tilde{y}-a_{1} \tilde{b} b_{0}^{T} y_{1}\right)$. Due to the inductive hypothesis, the origin of $\left.\dot{\tilde{y}}=\tilde{A}-\tilde{b} \tilde{\kappa}^{(1)}(0)\right) \tilde{y}$ is asymptotically stable. Thus, local asymptotic stability of (14) and (19) follows easily.

It remains to prove that systems (14) and (19) are $S I S S_{L}$. In both cases, using that $1-1 /(1+s)^{1 / 2} \leq s$ for all $s \geq 0$, it holds from (16) and (21) that

$$
\left\|g_{1}(\tilde{y})\right\| \leq \sum_{k=2}^{\mu+1} \theta_{1, k}\left\|y_{k}\right\|\left(\sum_{m=k}^{\mu+1}\left\|y_{m}\right\|^{2}\right) \leq\|\tilde{y}\|^{3} \sum_{k=2}^{\mu+1} \theta_{1, k},
$$

and from (15) and (20) that

$$
\left|\rho_{1}(y)\right| \leq\|\tilde{y}\|^{2} .
$$

Recall that, due to the inductive hypothesis, $\dot{\tilde{y}}=\tilde{A} \tilde{y}-\tilde{b} \tilde{k}(\tilde{y})$ is $\operatorname{SISS}_{L}(\tilde{\Delta}, \tilde{N})$ for some $\tilde{\Delta}>0$ and $\tilde{N}>0$. We next prove the $S I S S_{L}$ property for case ii).

Let

$$
\begin{aligned}
& C_{1}:=\tilde{N}\left(Q_{2, \mu+1}\|\tilde{b}\|+1\right), \\
& C_{2}:=C_{1}^{2}+C_{1}^{3} \sum_{k=2}^{\mu+1} \theta_{i, k} .
\end{aligned}
$$

From Lemma3 (with $\omega=\omega_{1}$ ), there exist $\Gamma_{1}, N_{1}>0$ such that for any $a_{1} \in(0,1]$ the system $\dot{y}_{1}=\omega_{1} A_{0} y_{1}-$ $a_{1} b_{0} \frac{b_{0}^{T} y_{1}}{\left(1+\left\|y_{1}\right\|^{2}\right)^{1 / 2}}$ is $\operatorname{SISS}_{L}\left(\Gamma_{1} a_{1}, N_{1} / a_{1}\right)$. Define

$$
\bar{a}_{1}:=\min \left\{1, \frac{\tilde{\Delta} \tilde{N}}{C_{1}}, \sqrt{\frac{\Gamma_{1}}{2 C_{2}}}, \sqrt{\frac{C_{1}}{4 Q_{2, \mu+1} \tilde{N}\|\tilde{b}\| N_{1} C_{2}}}\right\}
$$


and choose $a_{1} \in\left(0, \bar{a}_{1}\right]$. Let

$$
\Delta:=\min \left\{\frac{a_{1} \Gamma_{1}}{2}, a_{1}\right\} .
$$

Given $\delta \leq \Delta$, let $e_{1}: \mathbb{R}_{\geq 0} \rightarrow \mathbb{R}^{2}$ and $e_{2}: \mathbb{R}_{\geq 0} \rightarrow \mathbb{R}^{2 s+z-2}$ be two bounded measurable functions, eventually bounded by $\delta$. Consider any trajectory $\left(y_{1}(\cdot), \tilde{y}(\cdot)\right)$ of the following system

$$
\begin{aligned}
\dot{y}_{1} & =\omega_{1} A_{0} y_{1}-a_{1} b_{0} \frac{b_{0}^{T} y_{1}}{\left(1+\left\|y_{1}\right\|^{2}\right)^{1 / 2}}+a_{1} b_{0} \rho_{1}(y)+b_{0} g_{1}(\tilde{y})+e_{1}, \\
\dot{y} & =\tilde{A} \tilde{y}-\tilde{b} \tilde{\kappa}(\tilde{y})-\tilde{b} a_{1} f_{1}(y)+e_{2},
\end{aligned}
$$

In view of (19), (20), (21), (22) and (18) the above system is clearly forward complete. We next show that there exists a constant $N>0$ such that $\left\|y_{1}(t)\right\| \leq_{e v} N \delta$ and $\|\tilde{y}(t)\| \leq_{e v} N \delta$. From (22) and recalling that $\left\|b_{0}\right\|=1$, a straightforward computation yields

$$
\left\|a_{1} \tilde{b} f_{1}(y)\right\| \leq a_{1} Q_{2, \mu+1}\|\tilde{b}\|
$$

Since $\left\|e_{2}(t)\right\| \leq_{e v} \delta$, it follows that

$$
\left\|a_{1} \tilde{b} f_{1}(y(t))+e_{2}(t)\right\| \leq_{e v} a_{1} Q_{2, \mu+1}\|\tilde{b}\|+\delta
$$

Moreover from (27), (28) and it follows that

$$
\left\|a_{1} \tilde{b} f_{1}(y(t))+e_{2}(t)\right\| \leq_{e v} a_{1}\left(Q_{2, \mu+1}\|\tilde{b}\|+1\right) \leq a_{1} C_{1} / \tilde{N} \leq \tilde{\Delta},
$$

where $C_{1}$ is defined in $(25)$. Using the $\operatorname{SISS}_{L}(\tilde{\Delta}, \tilde{N})$ property of System $\dot{\tilde{y}}=\tilde{A} \tilde{y}-\tilde{b} \tilde{\kappa}(\tilde{y})$, it follows that the solution of 29) satisfies

$$
\|\tilde{y}(t)\| \leq_{e v} a_{1} C_{1} .
$$

Consequently, using (24) and (23), it follows that

$$
\left\|a_{1} b_{0} \rho_{1}(y(t))+b_{0} g_{1}(\tilde{y}(t))\right\| \leq_{e v} a_{1}^{3} C_{2} .
$$

Using (27), we have $a_{1}^{3} C_{2} \leq \frac{a_{1} \Gamma_{1}}{2}$. Moreover (28) ensures that $\left\|e_{1}(t)\right\| \leq \leq_{e v} \frac{a_{1} \Gamma_{1}}{2}$. So it follows that

$$
\left\|a_{1} b_{0} \rho_{1}(y(t))+b_{0} g_{1}(\tilde{y}(t))+e_{1}(t)\right\| \leq_{e v} a_{1} \Gamma_{1} .
$$

The $\operatorname{SISS}_{L}\left(\Gamma_{1} a_{1}, N_{1} / a_{1}\right)$ property of $\dot{y}_{1}=\omega_{1} A_{0} y_{1}-a_{1} b_{0} \frac{b_{0}^{T} y_{1}}{\left(1+\left\|y_{1}\right\|^{2}\right)^{1 / 2}}$ ensures that

$$
\left\|y_{1}(t)\right\| \leq_{e v} \frac{N_{1}}{a_{1}}\left(a_{1}^{3} C_{2}+\delta\right) \leq N_{1} \Gamma_{1}
$$

Now let $\theta>0$ be defined as

$$
\theta:=\limsup _{t \rightarrow+\infty}\|\tilde{y}(t)\| .
$$

Then $\|\tilde{y}(t)\| \leq_{e v} 2 \theta$. There are two cases to consider, either $2 \theta \leq a_{1} C_{1}$ or $a_{1} C_{1}<2 \theta$. In the case when $2 \theta \leq a_{1} C_{1}$, we have

$$
\left\|a_{1} b_{0} \rho_{1}(y(t))+b_{0} g_{1}(\tilde{y}(t))+e_{1}(t)\right\| \leq_{e v} 2 \theta a_{1}^{2} C_{2} / C_{1} .
$$


So invoking again the $\operatorname{SISS}_{L}\left(\bar{\rho}_{1} \Gamma_{1} a_{1}, N / a_{1}\right)$ property of $\dot{y}_{1}=\omega_{1} A_{0} y_{1}-a_{1} b_{0} \frac{b_{0}^{T} y_{1}}{\left(1+\left\|y_{1}\right\|^{2}\right)^{1 / 2}}$, one gets that the solution of 29] satisfies

$$
\left\|y_{1}(t)\right\| \leq_{e v} \frac{N_{1}}{a_{1}}\left(\frac{2 \theta a_{1}^{2} C_{2}}{C_{1}}+\delta\right) .
$$

In the case when $a_{1} C<2 \theta$, the estimate (33) follows readily from (31). Exploiting again the $\operatorname{SISS}_{L}(\tilde{\Delta}, \tilde{N})$ property of System $\dot{\tilde{y}}=\tilde{A} \tilde{y}-\tilde{b} \tilde{\kappa}(\tilde{y})$, it follows that

$$
\begin{aligned}
\|\tilde{y}(t)\| \quad \leq_{e v} \quad \tilde{N}\left(\|\tilde{b}\| Q_{2, \mu+1} N_{1}\left(\frac{2 \theta a_{1}^{2} C_{2}}{C_{1}}+\delta\right)+\delta\right) \\
=\quad \frac{2 Q_{2, \mu+1} \tilde{N}\|\tilde{b}\| N_{1} a_{1}^{2} C_{2}}{C_{1}}+\delta \tilde{N}\left(\|\tilde{b}\| Q_{2, \mu+1} N_{1}+1\right) .
\end{aligned}
$$

It then follows from (27) that

$$
\|\tilde{y}(t)\| \leq_{e v} \frac{\theta}{2}+\delta \tilde{N}\left(\|\tilde{b}\| Q_{2, \mu+1} N_{1}+1\right) .
$$

Taking the limsup of the above estimate, we get from (32) that

$$
\theta \leq 2 \delta \tilde{N}\left(\|\tilde{b}\| Q_{2, \mu+1} N_{1}+1\right)
$$

Consequently, we obtain that

$$
\begin{aligned}
\|\tilde{y}(t)\| \quad \leq_{e v} \quad 2 \tilde{N}\left(\|\tilde{b}\| Q_{2, \mu+1} N_{1}+1\right) \delta \\
\left\|y_{1}(t)\right\| \quad \leq_{e v} \quad 2 \frac{N_{1}}{a_{1}}\left(\frac{2 a_{1}^{2} C_{2}}{C_{1}}+1\right) \tilde{N}\left(N_{1}+1\right) \delta
\end{aligned}
$$

which finishes to establish $\left(P_{\mu+1}\right)$ for the case $\left.i i\right)$. Proceeding as in case $\left.i i\right)$, it can be shown that system 14 is $\operatorname{SISS}_{L}$. This end the inductive proof of $\left(P_{\mu}\right)$.

\subsubsection{Proof of Proposition 2}

Fix $\mu \in \mathbb{N}_{\geq 1}$. Let $s$ and $z$ be two integers such that $s+z=\mu, \omega_{1}, \ldots, \omega_{s}$ be positive constant numbers, and $a_{1}, \ldots, a_{\mu}$ be positive numbers less than or equal to 1 . Consider the system (7) with the feedback law (8), where $\mu(A)=\mu, s(A)=s$ and $z(A)=z$. We establish Proposition 2 by induction on $p$. More precisely we prove the following statement:

$\left(H_{p}\right)$ : For each $p \in \mathbb{N}$, there exist a positive constant $c_{\mu}$ and continuous functions $c_{i}: \mathbb{R}_{>0}^{\mu-i} \rightarrow \mathbb{R}_{>0}, i \in$ $\llbracket 1, \mu-1 \rrbracket$, such that for any trajectory $y(\cdot)$ of the closed-loop system (7) with the feedback law (8), the control signal $U: \mathbb{R}_{\geq 0} \rightarrow \mathbb{R}$ defined by $U(t):=\kappa(y(t))$ for all $t \geq 0$ satisfies, for all $k \in \llbracket 0, p \rrbracket$,

$$
\left|U^{(k)}(t)\right| \leq a_{\mu} c_{\mu}+\sum_{i=1}^{\mu-1} a_{i} c_{i}\left(a_{\mu}, \ldots, a_{i+1}\right), \quad \forall t \geq 0
$$


For $p=0$, this statement $\left(H_{0}\right)$ holds trivially. Indeed, it is easy to see that for any trajectory of the closed-loop system (7) with the feedback law (8) we have

$$
|U(t)| \leq a_{\mu}+\sum_{i=1}^{\mu-1} a_{i} Q_{i+1, \mu}, \quad \forall t \geq 0 .
$$

Now, assume that $\left(H_{p}\right)$ holds true for some $p \in \mathbb{N}$. We next prove that $\left(H_{p+1}\right)$ also holds true. To that aim, let $y(\cdot)$ be any trajectory of the closed-loop system (7) with the feedback law (8), and the control signal $U(t):=\kappa(y(t)), \forall t \geq 0$. By the induction hypothesis, there exist a positive constant $\Upsilon_{\mu}$ and continuous functions $\Upsilon_{i}: \mathbb{R}_{>0}^{\mu-i} \rightarrow \mathbb{R}_{>0}, i \in \llbracket 1, \mu-1 \rrbracket$, such that for every $k \in \llbracket 0, p \rrbracket$ it holds that

$$
\left|U^{(k)}(t)\right| \leq a_{\mu} \Upsilon_{\mu}+\sum_{i=1}^{\mu-1} a_{i} \Upsilon_{i}\left(a_{\mu}, \ldots, a_{i+1}\right), \quad \forall t \geq 0
$$

It is sufficient to show that there exist a positive constant $\tilde{\Upsilon}_{\mu}$ and continuous functions $\tilde{\Upsilon}_{i}: \mathbb{R}_{>0}^{\mu-i} \rightarrow \mathbb{R}_{>0}$, $i \in \llbracket 1, \mu-1 \rrbracket$, such that

$$
\left|U^{(p+1)}(t)\right| \leq a_{\mu} \tilde{\Upsilon}_{\mu}+\sum_{i=1}^{\mu-1} a_{i} \tilde{\Upsilon}_{i}\left(a_{\mu}, \ldots, a_{i+1}\right), \quad \forall t \geq 0
$$

Indeed, the desired results will be obtained by setting $c_{\mu}:=\max \left\{\Upsilon_{\mu}, \tilde{\Upsilon}_{\mu}\right\}$, and $c_{i}(\cdot):=\max \left\{\Upsilon_{i}(\cdot), \tilde{\Upsilon}_{i}(\cdot)\right\}$ for $i \in \llbracket 1, \mu-1 \rrbracket$. In order to establish $[35$, we start by defining the following auxiliary functions:

$$
g(s):=s^{-1 / 2}, \quad \forall s>0
$$

and, for all $t \geq 0$,

$$
f_{i}(t):=\quad 1+\sum_{l=i}^{\mu}\left\|y_{l}(t)\right\|^{2}, \quad i \in \llbracket 1, \mu \rrbracket .
$$

Then, we can rewrite $U(\cdot)$ as

$$
U(t)=-\sum_{i=1}^{\mu} U_{i}(t), \quad \forall t \geq 0
$$

where, for every $i \in \llbracket 1, \mu \rrbracket$,

$$
U_{i}(t):=Q_{i, \mu} b_{0, i}^{T} y_{i}(t) g\left(f_{i}(t)\right), \quad \forall t \geq 0,
$$

where $b_{0, i}=b_{0}$ for all $i \in \llbracket 1, s \rrbracket$ and $b_{0, i}=1$ otherwise, and $Q_{i, \mu}$ is defined in (9). The $(p+1)$-th time derivative of the control signal $U(\cdot)$ is given, for all $t \geq 0$, by $U^{(p+1)}(t)=-\sum_{i=1}^{\mu} U_{i}^{(p+1)}(t)$. Therefore to prove $\left(H_{p+1}\right)$, it is sufficient to show that, for each $i \in \llbracket 1, \mu \rrbracket$, there exists continuous functions $c_{i, l}: \mathbb{R}_{>0}^{\mu-l} \rightarrow$ $\mathbb{R}_{>0}, l \in \llbracket 1, i \rrbracket$, such that, for all $t \geq 0$,

$$
\left|U_{i}^{(p+1)}(t)\right| \leq \sum_{l=1}^{i} a_{l} c_{i, l}\left(a_{\mu}, \ldots, a_{l+1}\right),
$$

$c_{i, \mu}$ is actually a constant independent of $a_{\mu}$, we write it as $c_{i, \mu}\left(a_{\mu}, a_{\mu+1}\right)$ for the sake of notation homogeneity. 
For $i \in \llbracket 1, \mu \rrbracket$, we apply Leibniz's rule to $[39]$ with respect to $b_{0, i}^{T} y_{i}(t)$ and $g\left(f_{i}(t)\right)$ and obtain that the $(p+1)$-th time derivative of $U_{i}(\cdot)$ is given, for all $t \geq 0$, by

$$
U_{i}^{(p+1)}(t)=a_{i} Q_{i+1, \mu}\left(\sum_{l_{1}=0}^{p+1}\left(\begin{array}{c}
p+1 \\
l_{1}
\end{array}\right) b_{0, i}^{T} y_{i}^{\left(p+1-l_{1}\right)}(t)\left[g \circ f_{i}\right]^{\left(l_{1}\right)}(t)\right) .
$$

To obtain (40), it is sufficient to prove that for each $i \in \llbracket 1, \mu \rrbracket$, and $l_{1} \in \llbracket 0, p+1 \rrbracket$ there exist continuous functions $\beta_{i, l, l_{1}}: \mathbb{R}_{>0}^{\mu-l} \rightarrow \mathbb{R}_{>0}$ for $l \in \llbracket 1, i \rrbracket$ such that, for all $t \geq 0$,

$$
\left|b_{0, i}^{T} y_{i}^{\left(p+1-l_{1}\right)}(t)\left[g \circ f_{i}\right]^{\left(l_{1}\right)}(t)\right| \leq \beta_{i, i, l_{1}}\left(a_{\mu}, \ldots, a_{i+1}\right)+\sum_{l=1}^{i-1} a_{l} \beta_{i, l, l_{1}}\left(a_{\mu}, \ldots, a_{l+1}\right) .
$$

In order to get (41) we next provide, for each $i \in \llbracket 1, \mu \rrbracket$, estimates of $\left\|y_{i}^{\left(l_{1}\right)}(t)\right\|,\left|f_{i}^{\left(l_{1}\right)}(t)\right|$ and $\left[g \circ f_{i}\right]^{\left(l_{1}\right)}(t)$ for $l_{1} \in \llbracket 1, p+1 \rrbracket$. One can observe that, for each $i \in \llbracket 1, \mu \rrbracket, \dot{y}_{i}$ depends on the constants $a_{i+1}, \ldots, a_{\mu}$, the states $y_{i}, \ldots, y_{\mu}$ and the feedback $u=\kappa(y)$. By an induction argument using differentiation of system (7), one can obtain the following statement: for any $k \in \llbracket 1, p+1 \rrbracket, i \in \llbracket 1, \mu \rrbracket$, there exist continuous functions

$$
\Psi_{k, i, l} \quad: \quad \mathbb{R}_{>0}^{\mu-i} \rightarrow \mathbb{R}_{>0}, \quad l \in \llbracket i+1, \mu \rrbracket, \quad \Phi_{k, i, l}: \mathbb{R}_{>0}^{\mu-i} \rightarrow \mathbb{R}_{>0}, \quad l \in \llbracket 0, p \rrbracket,
$$

such that, for all positive times, it holds that

$$
\left\|y_{i}^{(k)}(t)\right\| \leq \sum_{l=i}^{\mu} \Psi_{k, i, l}\left(a_{\mu}, \ldots, a_{i+1}\right)\left\|y_{l}(t)\right\|+\sum_{l=0}^{k-1} \Phi_{k, i, l}\left(a_{\mu}, \ldots, a_{i+1}\right)\left|U^{(l)}(t)\right|,
$$

where, by convention, $\Psi_{k, i, \mu}$ are constant functions independent of $a_{\mu}$ for $k \in \llbracket 1, p+1 \rrbracket$ and $i \in \llbracket 1, \mu \rrbracket$. Using (34) in the above estimate, one gets that, for any $k \in \llbracket 1, p+1 \rrbracket$ and $i \in \llbracket 1, \mu-1 \rrbracket$, there exist functions $\tilde{v}_{l, k, i}: \mathbb{R}_{>0}^{\mu-i} \rightarrow \mathbb{R}_{>0}$, for $l \in \llbracket i+1, \mu \rrbracket$, and $\tilde{\Phi}_{l, k, i}: \mathbb{R}_{>0}^{\mu-i} \rightarrow \mathbb{R}_{>0}$ such that, for all $t \geq 0$,

$$
\left\|y_{i}^{(k)}(t)\right\| \leq \sum_{l=i}^{\mu} \Psi_{k, i, l}\left(a_{\mu}, \ldots, a_{i+1}\right)\left\|y_{l}(t)\right\|+\tilde{\Phi}_{k, i}\left(a_{\mu}, \ldots, a_{i+1}\right)+\sum_{l=1}^{i} a_{l} \tilde{v}_{l, k, i}\left(a_{\mu}, \ldots, a_{l+1}\right) .
$$

Setting, for $i \in \llbracket 1, \mu \rrbracket$,

$$
\begin{aligned}
\bar{\Psi}_{i}\left(a_{\mu}, \ldots, a_{i+1}\right) & :=\max \left\{\Psi_{k, i, l}\left(a_{\mu}, \ldots, a_{i+1}\right): k \in \llbracket 1, p+1 \rrbracket, l \in \llbracket i+1, \mu \rrbracket\right\}, \\
\bar{\Phi}_{i}\left(a_{\mu}, \ldots, a_{i+1}\right) & :=\max \left\{\tilde{\Phi}_{k, i}\left(a_{\mu}, \ldots, a_{i+1}\right): k \in \llbracket 1, p+1 \rrbracket\right\}, \\
\tilde{v}_{l, i}\left(a_{\mu}, \ldots, a_{l+1}\right) & :=\max \left\{\tilde{v}_{l, k, i}\left(a_{\mu}, \ldots, a_{l+1}\right): k \in \llbracket 1, p+1 \rrbracket\right\}, \quad l \in \llbracket 1, i \rrbracket,
\end{aligned}
$$

one can obtain that, for all $k \in \llbracket 1, p+1 \rrbracket$, all $i \in \llbracket 1, \mu \rrbracket$, and all $t \geq 0$,

$$
\left\|y_{i}^{(k)}(t)\right\| \leq \bar{\Psi}_{i}\left(a_{\mu}, \ldots, a_{i+1}\right) \sum_{l=i}^{\mu}\left\|y_{l}(t)\right\|+\bar{\Phi}_{i}\left(a_{\mu}, \ldots, a_{i+1}\right)+\sum_{l=1}^{i} a_{l} \tilde{v}_{l, i}\left(a_{\mu}, \ldots, a_{l+1}\right) .
$$

It follows that (41) for $l_{1}=0$ holds true. For any $i \in \llbracket 1, \mu \rrbracket$ and $k \in \llbracket 1, p+1 \rrbracket$, the $k$-th time derivative of $f_{i}(\cdot)$, defined in (37), is given, for all $t \geq 0$, by

$$
f_{i}^{(k)}(t)=\sum_{l_{1}=0}^{k}\left(\begin{array}{c}
k \\
l_{1}
\end{array}\right) \sum_{l_{2}=i}^{\mu}\left(y_{l_{2}}^{\left(l_{1}\right)}(t)\right)^{T} y_{l_{2}}^{\left(k-l_{1}\right)}(t)
$$


Thus, one can get that

$$
\begin{aligned}
\left|f_{i}^{(k)}(t)\right| & \leq 2 \sum_{l_{2}=i}^{\mu}\left\|y_{l_{2}}(t)\right\|\left\|y_{l_{2}}^{(k)}(t)\right\|+\sum_{l_{1}=1}^{k-1}\left(\begin{array}{c}
k \\
l_{1}
\end{array}\right) \sum_{l_{2}=i}^{\mu}\left\|y_{l_{2}}^{\left(l_{1}\right)}(t)\right\|\left\|y_{l_{2}}^{\left(k-l_{1}\right)}(t)\right\|, \\
& \leq \sum_{l_{2}=i}^{\mu}\left(\left\|y_{l_{2}}(t)\right\|^{2}+\left\|y_{l_{2}}^{(k)}(t)\right\|^{2}\right)+\sum_{l_{1}=1}^{k-1}\left(\begin{array}{c}
k \\
l_{1}
\end{array}\right) \sum_{l_{2}=i}^{\mu}\left(\left\|y_{l_{2}}^{\left(l_{1}\right)}(t)\right\|^{2}+\left\|y_{l_{2}}^{\left(k-l_{1}\right)}(t)\right\|^{2}\right) .
\end{aligned}
$$

From (42), and using the fact that $\left(\sum_{i_{1}=1}^{m}\left|x_{i_{1}}\right|\right)^{2} \leq m \sum_{i_{1}=1}^{m} x_{i_{1}}^{2}$, one can obtain that for each $l_{2} \in \llbracket 1, \mu \rrbracket$ and $l_{1} \in \llbracket 1, p+1 \rrbracket$ it holds that, for all $t \geq 0$,

$$
\left\|y_{l_{2}}^{\left(l_{1}\right)}(t)\right\|^{2} \leq(\mu+2)\left(\bar{\Psi}_{l_{2}}\left(a_{\mu}, \ldots, a_{l_{2}+1}\right)^{2} \sum_{l=l_{2}}^{\mu}\left\|y_{l}(t)\right\|^{2}+\bar{\Phi}_{l_{2}}\left(a_{\mu}, \ldots, a_{l_{2}+1}\right)^{2}+\sum_{l=1}^{l_{2}}\left(a_{l} \tilde{v}_{l, l_{2}}\left(a_{\mu}, \ldots, a_{l+1}\right)\right)^{2}\right) .
$$

Since the right-hand side of (43) is independent of $l_{1}$, and $a_{l} \leq 1$ for all $l \in \llbracket 1, \mu \rrbracket$, one can gets that there exist continuous functions

$$
\begin{array}{rlll}
\tilde{\Psi}_{l} & : & \mathbb{R}_{>0}^{\mu-l} \rightarrow \mathbb{R}_{>0}, & l \in \llbracket 1, \mu \rrbracket, \\
\tilde{\Phi}_{l} & : & \mathbb{R}_{>0}^{\mu-l} \rightarrow \mathbb{R}_{>0}, \quad l \in \llbracket 1, \mu \rrbracket, \\
\tilde{\bar{v}}_{l, l_{1}} & : & \mathbb{R}_{>0}^{\mu-l} \rightarrow \mathbb{R}_{>0}, & l_{1} \in \llbracket 1, \mu \rrbracket, l \in \llbracket 1, l_{1} \rrbracket,
\end{array}
$$

such that, for any $k \in \llbracket 1, p \rrbracket$ and all $t \geq 0$, it holds

$$
\left|f_{i}^{(k)}(t)\right| \leq \tilde{\bar{\Psi}}_{l_{2}}\left(a_{\mu}, \ldots, a_{i+1}\right) \sum_{l=i}^{\mu}\left\|y_{l}(t)\right\|^{2}+\tilde{\bar{\Phi}}_{l_{2}}\left(a_{\mu}, \ldots, a_{i+1}\right)+\sum_{l=1}^{i} a_{l} \tilde{\bar{v}}_{l, i}\left(a_{\mu}, \ldots, a_{l+1}\right) .
$$

A trivial estimate for any $k \in \llbracket 1, p+1 \rrbracket$, any $i \in \llbracket 1, \mu \rrbracket$, and all $t \geq 0$ is given by

$$
\left|f_{i}^{(k)}(t)\right| \leq \tilde{\Psi}_{i}\left(a_{\mu}, \ldots, a_{i+1}\right) f_{i}(t)+\tilde{\bar{\Phi}}_{i}\left(a_{\mu}, \ldots, a_{i+1}\right)+\sum_{l=1}^{i} a_{l} \tilde{\bar{v}}_{l, l_{2}}\left(a_{\mu}, \ldots, a_{l+1}\right) .
$$

By the Faà di Bruno's formula (given in Lemma 5 in Appendix), for each $i \in \llbracket 1, \mu \rrbracket$, and $l_{1} \in \llbracket 1, p+1 \rrbracket$, the $l_{1}$-th time derivative of $g \circ f_{i}(\cdot)$ is given, for all $t \geq 0$, by

$$
\left[g \circ f_{i}\right]^{\left(l_{1}\right)}(t)=\sum_{l_{2}=1}^{l_{1}} g^{\left(l_{2}\right)}\left(f_{i}(t)\right) \sum_{\delta \in \mathscr{P}_{l_{1}, l_{2}}} c_{\delta} \prod_{l=1}^{l_{1}-l_{2}+1}\left(f_{i}^{(l)}(t)\right)^{\delta_{l}}
$$

where $\mathscr{P}_{l_{1}, l_{2}}$ denotes the set of $\left(l_{1}-l_{2}+1\right)$-tuples $\delta:=\left(\delta_{1}, \delta_{2}, \ldots, \delta_{l_{1}-l_{2}+1}\right)$ of positive integers satisfying $\delta_{1}+\delta_{2}+\ldots+\delta_{l_{1}-l_{2}+1}=l_{2}$ and $\delta_{1}+2 \delta_{2}+\ldots+\left(l_{1}-l_{2}+1\right) \delta_{l_{1}-l_{2}+1}=l_{1}$. Observe that the $k$-th derivative of the function $g$ defined in (36) reads

$$
g^{(k)}(s)=d_{k} s^{-1 / 2-k}, \quad \forall s>0,
$$

with $d_{k}=(-1)^{k} \prod_{l=0}^{k-1}(1 / 2+l)$. Using (45), and taking the absolute value, one can get, for all $t \geq 0$,

$$
\left|\left[g \circ f_{i}\right]^{\left(l_{1}\right)}(t)\right| \leq \sum_{l_{2}=1}^{l_{1}} d_{l_{2}} \frac{1}{\left(f_{i}(t)\right)^{l_{2}+1 / 2}} \sum_{\delta \in \mathscr{P}_{l_{1}, l_{2}}} c_{\delta} \prod_{l=1}^{l_{1}-l_{2}+1}\left|f_{i}^{(l)}(t)\right|^{\delta_{l}} .
$$


Using (44), one can obtain that, for any $l_{1} \in \llbracket 1, p+1 \rrbracket$, any $l_{2} \in \llbracket 1, l_{1} \rrbracket$ and for all $t \geq 0$,

$$
\begin{aligned}
\sum_{\delta \in \mathscr{P}_{l_{1}, l_{2}}} c_{\delta} \prod_{l=1}^{l_{1}-l_{2}+1}\left|f_{i}^{(l)}(t)\right|^{\delta_{l}} \leq & \left(\tilde{\bar{\Psi}}_{i}\left(a_{\mu}, \ldots, a_{i+1}\right) f_{i}(t)+\tilde{\bar{\Phi}}_{i}\left(a_{\mu}, \ldots, a_{i+1}\right)\right. \\
& \left.+\sum_{l_{3}=1}^{i} a_{l_{3}} \tilde{\bar{v}}_{l_{3}, i}\left(a_{\mu}, \ldots, a_{i+1}\right)\right)^{l_{2}} \sum_{\delta \in \mathscr{P}_{l_{1}, l_{2}}} c_{\delta} .
\end{aligned}
$$

It follows that, for all $l_{1} \in \llbracket 1, p+1 \rrbracket, t \geq 0$,

$$
\begin{aligned}
\left|\left[g \circ f_{i}\right]^{\left(l_{1}\right)}(t)\right| \leq & \sum_{l_{2}=1}^{l_{1}} d_{l_{2}} \frac{\delta \in \sum_{l_{1}, l_{2}} c_{\delta}}{\left(f_{i}(t)\right)^{1 / 2}}( \\
& \left.\frac{\tilde{\Psi}_{i}\left(a_{\mu}, \ldots, a_{i+1}\right) f_{i}(t)+\tilde{\Phi}_{i}\left(a_{\mu}, \ldots, a_{i+1}\right)+\sum_{l_{3}=1}^{i} a_{l_{3}} \tilde{\bar{v}}_{l_{3}, i}\left(a_{\mu}, \ldots, a_{i+1}\right)}{f_{i}(t)}\right)^{l_{2}}, \\
\leq & \sum_{l_{2}=1}^{l_{1}} d_{l_{2}} \frac{\delta \in \mathscr{P}_{l_{1}, l_{2}}}{\left(f_{i}(t)\right)^{1 / 2}}( \\
& \left.\tilde{\bar{\Psi}}_{i}\left(a_{\mu}, \ldots, a_{i+1}\right)+\tilde{\bar{\Phi}}_{i}\left(a_{\mu}, \ldots, a_{i+1}\right)+\sum_{l_{3}=1}^{i} a_{l_{3}} \tilde{\bar{v}}_{l_{3}, i}\left(a_{\mu}, \ldots, a_{i+1}\right)\right)^{l_{2}},
\end{aligned}
$$

Thus, it can be seen that, for every $i \in \llbracket 1, \mu \rrbracket$ and $l_{1} \in \llbracket 1, p+1 \rrbracket$, there exist continuous functions $\Gamma_{i, l_{1}}$ : $\mathbb{R}_{>0}^{\mu-i} \rightarrow \mathbb{R}_{>0}$ and $\Gamma_{i, l_{1}, l}: \mathbb{R}_{>0}^{\mu-l} \rightarrow \mathbb{R}_{>0}, l \in \llbracket 1, i+1 \rrbracket$, such that, for all $t \geq 0$,

$$
\left|\left[g \circ f_{i}\right]^{\left(l_{1}\right)}(t)\right| \leq \frac{1}{\left(f_{i}(t)\right)^{1 / 2}}\left(\Gamma_{i, l_{1}}\left(a_{\mu}, \ldots, a_{i+1}\right)+\sum_{l=1}^{i} a_{i} \Gamma_{i, l_{1}, l}\left(a_{\mu}, \ldots, a_{i+1}\right)\right) .
$$

Then, from (46) and (42) it follows that (41) holds true for any $l_{1} \in \llbracket 1, p+1 \rrbracket$. This ends the inductive proof of $\left(H_{p}\right)$.

\subsection{Proof of Theorem 2}

\subsubsection{Reduction of the proof of Theorem 2 to the proof of Propositions 1 and 3}

We prove Theorem 2 by induction on the number of inputs $q$. We show that the inductive step reduces to Proposition 1 and Proposition 3 which is proven in Section 3.2.2

For $q=1$, the conclusion follows from Theorem 1 For a given $q \in \mathbb{N}_{\geq 1}$ assume that Theorem 2 holds. We show that Theorem 2 then holds for LTI systems given in the reduced controllability form with $q+1$ inputs. Let $p \in \mathbb{N}$ and $\left(R_{j}\right)_{0 \leq j \leq p}$ be a $(p+1)$-tuple of positive real numbers. Define $\underline{R}:=\min _{j \in \llbracket 0, p \rrbracket} R_{j}$. 
Given $n \in \mathbb{N}_{\geq 2}$ consider a LTI system given in the reduced controllability form with $\tilde{q}:=q+1$ inputs by

$$
\begin{array}{rrr}
\dot{x}_{0}= & A_{00} x_{0}+A_{01} x_{1}+A_{02} x_{2}+\ldots+A_{0 \tilde{q}} x_{\tilde{q}}+ & b_{01} u_{1}+b_{02} u_{2}+\ldots+b_{0 \tilde{q}} u_{\tilde{q}}, \\
\dot{x}_{1}= & A_{11} x_{1}+A_{12} x_{2}+\ldots+A_{1 \tilde{q}} x_{\tilde{q}}+ & b_{11} u_{1}+b_{22} u_{2}+\ldots+b_{1 \tilde{q}} u_{\tilde{q}}, \\
\dot{x}_{2}= & A_{22} x_{2}+\ldots+A_{2 \tilde{q}} x_{\tilde{q}}+ & b_{22} u_{2}+\ldots+b_{2 \tilde{q}} u_{\tilde{q}}, \\
\vdots & & \\
\dot{x}_{\tilde{q}}= & A_{\tilde{q} \tilde{q}} x_{\tilde{q}}+ & \\
& & b_{\tilde{q} \tilde{q}} u_{\tilde{q}},
\end{array}
$$

where $x_{i} \in \mathbb{R}^{n_{i}}$ and $u_{i} \in \mathbb{R}$ for each $i \in \llbracket 0, q+1 \rrbracket, A_{00}$ is Hurwitz, for every $i \in \llbracket 1, q+1 \rrbracket$ all the eigenvalues of $A_{i i}$ are critical, and the pairs $\left(A_{i i}, b_{i i}\right)$ are controllable.

Since $A_{00}$ is Hurwitz, if we find a feedback law $p$-bounded by $\left(R_{j}\right)_{0 \leq j \leq p}$, and $S I S S_{L}$-stabilizing for $\left(x_{1}, \ldots, x_{q+1}\right)$-subsystem then, clearly, this feedback does the job for the complete system. From now on, we only consider the $\left(x_{1}, \ldots, x_{q+1}\right)$-subsystem and we rewrite it compactly as

$$
\begin{aligned}
\dot{x}_{1} & =A_{11} x_{1}+b_{11} u_{1}+\tilde{A} z+\tilde{B} \bar{u}, \\
\dot{z} & =\bar{A} z+\bar{B} \bar{u},
\end{aligned}
$$

where $z:=\left[x_{2}, \ldots, x_{q+1}\right]^{T}, u:=\left[u_{2}, \ldots, u_{q+1}\right]^{T}$.

We next provide a key technical lemma.

Lemma 4. Let $\dot{x}=A x+b u, x \in \mathbb{R}^{n}, u \in \mathbb{R}$, be a controllable single input linear system. Assume that all the eigenvalues of $A$ are critical. Let $\pm i \omega_{1}, \ldots, \pm i \omega_{s(A)}$ be the nonzero eigenvalues of $A,\left(a_{2}, \ldots, a_{\mu(A)}\right)$ be a sequence of positive numbers and $T \in \mathbb{R}^{n, n}$ be such that the linear change of coordinate $y=T x$ transforms $\dot{x}=A x+$ bu into system (7) compactly written as $\dot{y}=J y+b u$. Rewrite $T$ as

$$
T=\left[T_{1}, \ldots, T_{s(A)}, T_{s(A)+1}, \ldots, T_{\mu(A)}\right]^{T},
$$

where $T_{i} \in \mathbb{R}^{2, n}$ if $i \in \llbracket 1, s(A) \rrbracket$ otherwise $T_{i} \in \mathbb{R}^{1, n}$. Then $T$ has the following property

$(\mathscr{I}): T_{\mu(A)}$ is independent of $\left(a_{2}, \ldots, a_{\mu(A)}\right)$, and each $T_{i}$ depend only on $\left(a_{i+1}, \ldots, a_{\mu(A)}\right)$.

Moreover, given $r, k \in \mathbb{N}$, let $M \in \mathbb{R}^{n, r}$ be independent of the constants $a_{i}$, then the matrices $T M$ and $J^{k} T$ satisfy property $(\mathscr{I})$.

The proof of Lemma 4 follows from a careful examination of the proofs of Lemmas 3.1 and 5.1 in [6].

Let $\left(a_{2}, \ldots, a_{\mu\left(A_{11}\right)}\right)$ be a sequence of positive numbers (to be chosen later). Let $T$ be the linear change of coordinate that transforms $\dot{x}=A_{11} x+b_{11} u_{1}$ into the form of system (7) compactly written as $\dot{y}=J y+b u$. We now make the following changes of coordinates $y=T x$, and system (47) is then given by

$$
\begin{aligned}
& \dot{y}=J y+b u_{1}+T \tilde{A} z+T \tilde{B} \bar{u}, \\
& \dot{z}=\bar{A} z+\bar{B} \bar{u} .
\end{aligned}
$$

Let $\kappa$ be a feedback law $p$-bounded feedback law by $\left(R_{j} / 2\right)_{0 \leq j \leq p}$, and $\operatorname{SISS}_{L}\left(N_{2}, \Delta_{2}\right)$-stabilizing for subsystem (48b), for some $N_{2}, \Delta_{2}>0$ (thanks to the inductive hypothesis, we know that this feedback exists). Let $a_{1}>0$, to be chosen later. We seek the following feedback:

$$
\begin{aligned}
u_{1}(y, z) & :=\frac{\mu(y)}{\left(1+\|z\|^{2}\right)^{p}}, \\
\bar{u}(z) & :=\kappa(z),
\end{aligned}
$$


where $\mu(y)$ is defined in $[8]$. We now show that there exist positive constants $\left(a_{1}, a_{2}, \ldots, a_{\mu\left(A_{11}\right)}\right)$ such that the feedback law (49) is a feedback law $p$-bounded and $S I S S_{L}$-stabilizing for system (48). This choice is based on Proposition 1 and the following statement which is proven in Section 3.2.2

Proposition 3 ( $p$-bounded feedback). Let $a_{i}$, for $i \in \llbracket 1, \mu\left(A_{11}\right) \rrbracket$, be positive constants in $(0,1]$. Consider system (48) with the feedback law (49). Assume that $\kappa$ is a feedback law $p$-bounded by $\left(R_{j} / 2\right)_{0 \leq j \leq p}$, and $\operatorname{SISS}_{L}\left(N_{2}, \Delta_{2}\right)$-stabilizing for subsystem (48b). Then, there exist a positive constant $c_{\mu\left(A_{11}\right)}$, and continuous functions $c_{i}: \mathbb{R}_{>0}^{\mu\left(A_{11}\right)-i} \rightarrow \mathbb{R}_{>0}, i \in \llbracket 1, \mu\left(A_{11}\right)-1 \rrbracket$, such that for any trajectory of the closed-loop system (48) with the feedback law (49), the control signal $U_{1}: \mathbb{R}_{\geq 0} \rightarrow \mathbb{R}$ defined by $U_{1}(t):=u_{1}(y(t), z(t))$ for all $t \geq 0$ satisfies, for all $k \in \llbracket 0, p \rrbracket$,

$$
\left|U_{1}^{(k)}(t)\right| \leq a_{\mu} c_{\mu\left(A_{11}\right)}+\sum_{i=1}^{\mu\left(A_{11}\right)-1} a_{i} c_{i}\left(a_{\mu\left(A_{11}\right)}, \ldots, a_{i+1}\right), \quad \forall t \geq 0 .
$$

Pick $a_{\mu\left(A_{11}\right)} \in(0,1]$ in such a way that

$$
a_{\mu\left(A_{11}\right)} \leq \frac{\underline{R}}{2(p+1) c_{\mu\left(A_{11}\right)}} .
$$

Choose recursively $a_{i} \in(0,1], i=\mu\left(A_{11}\right)-1, \ldots, 1$, such that

$$
a_{i} \leq \bar{a}_{i}\left(a_{i+1}\right), \quad a_{i} \leq \frac{\underline{R}}{2(p+1) c_{i}\left(a_{\mu(A)}, \ldots, a_{i+1}\right)},
$$

where the functions $c_{i}$ appearing above are defined in Proposition 3 and the functions $\bar{a}_{i}$ are defined in

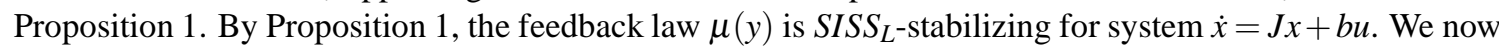
prove that the closed-loop system (48) with the feedback (49) is $S I S S_{L}$ (now, all the coefficients have been chosen). To that aim, first notice that there exist $\alpha_{1}, \alpha_{2}>0$ such that, for all $\|z\| \leq 1$,

$$
\begin{aligned}
\|T \tilde{A} z+T \tilde{B} \kappa(z)\| & \leq \alpha_{1}\|z\|, \\
\left\|b \mu(y)\left(1-\frac{1}{\left(1+\|z\|^{2}\right)^{p}}\right)\right\| & \leq \alpha_{2}\|z\| .
\end{aligned}
$$

Let

$$
\Delta:=\min \left\{1, \Delta_{2}, \frac{1}{N_{2}}, \frac{\Delta_{1}}{\left(\alpha_{2}+\alpha_{1}\right) N_{2}+1}\right\} .
$$

Given $\delta \leq \Delta$, let $e_{1}, e_{2}$ be two bounded measurable functions of the appropriate dimension, eventually bounded by $\delta$. Consider any trajectory $(y(\cdot), z(\cdot))$ of the following system

$$
\begin{aligned}
& \dot{y}=J y+b \mu(y)-b \mu(y)\left(1-\frac{1}{\left(1+\|z\|^{2}\right)^{p}}\right)+T \tilde{A} z+T \tilde{B} \kappa(z)+e_{1}, \\
& \dot{z}=\bar{A} z+\bar{B} \kappa(z)+e_{2},
\end{aligned}
$$

From the $\operatorname{SISS}_{L}\left(\Delta_{2}, N_{2}\right)$ property of $z$-subsystem it follows that $\|z(t)\| \leq_{e v} N_{2} \delta \leq 1$. Thus, using the above estimate, it is immediate to see that

$$
\left\|b \mu(y(t))\left(1-\frac{1}{\left(1+\|z(t)\|^{2}\right)^{p}}\right)+T \tilde{A} z(t)+T \tilde{B} \kappa(z(t))+e_{1}(t)\right\| \leq_{e v} \delta\left(\left(\alpha_{1}+\alpha_{2}\right) N_{2}+1\right) \leq \Delta_{1} .
$$


Therefore, invoking the $\operatorname{SISS}_{L}\left(\Delta_{1}, N_{1}\right)$ property of $\dot{x}=J x+b \mu(y)$, it follows that $\|y(t)\| \leq_{e v} \delta\left(\left(\alpha_{1}+\alpha_{2}\right) N_{2}+\right.$ 1) $N_{1}$. So, the closed-loop system (48) with the feedback (49) is $S I S S_{L}$. Moreover, as a consequence of Proposition 3 and of the inductive hypothesis, for any trajectory of the closed-loop system (7) with the feedback law (49), the control signal $U: \mathbb{R}_{\geq 0} \rightarrow \mathbb{R}^{m}$, defined by $U(\cdot):=\left[U_{1}(\cdot), U_{2}(\cdot)\right]^{T}$ with $U_{1}(t):=u_{1}(y(t), z(t))$ and $U_{2}(t):=\kappa(z(t))$ for all $t \geq 0$, satisfies

$$
\sup _{t \geq 0}\left\|U^{(k)}(t)\right\| \leq R_{k}
$$

for all $k \in \llbracket 0, p \rrbracket$. Thus, the feedback law (49) is a feedback law $p$-bounded by $\left(R_{j}\right)_{0 \leq j \leq p}$ for system (48).

\subsubsection{Proof of Proposition 3}

For the sake of notation compactness let $\mu=\mu\left(A_{11}\right)$. To prove Proposition 3, we establish by induction on $k$ that the following property holds, for all $k \in \llbracket 0, p \rrbracket$ :

$\left(\bar{H}_{k}\right)$ : There exist a positive constant $c_{\mu}$, and continuous functions $c_{i}: \mathbb{R}_{>0}^{\mu-i} \rightarrow \mathbb{R}_{>0}, i \in \llbracket 1, \mu-1 \rrbracket$, such that for any trajectory of the closed-loop system (48) with the feedback law (49), the control signal $U_{1}: \mathbb{R}_{\geq 0} \rightarrow \mathbb{R}$ defined by $U_{1}(t):=u_{1}(y(t), z(t))$ for all $t \geq 0$ satisfies, for all $j \in \llbracket 0, k \rrbracket$,

$$
\left|U_{1}^{(j)}(t)\right| \leq a_{\mu} c_{\mu}+\sum_{i=1}^{\mu-1} a_{i} c_{i}\left(a_{\mu}, \ldots, a_{i+1}\right), \quad \forall t \geq 0 .
$$

For $k=0$, the statement $\left(\bar{H}_{0}\right)$ holds trivially. Now, assume that $\left(\bar{H}_{k}\right)$ holds true for some $k \in \llbracket 0, p-1 \rrbracket$. We next prove that $\left(\bar{H}_{k+1}\right)$ also holds true. Let $(y(\cdot), z(\cdot))$ be any trajectory of the closed-loop system (48) with the feedback law (49), and the control signal $\left.U_{1}(t):=u_{1}(y(t), z(t))\right)$ and $U_{2}(t):=\kappa(z(t)), \forall t \geq 0$. As in the proof of Proposition 2] it is sufficient to prove that there exist a positive constant $\tilde{\Upsilon}_{\mu}$ and continuous functions $\tilde{\Upsilon}_{i}: \mathbb{R}_{>0}^{\mu-i} \rightarrow \mathbb{R}_{>0}, i \in \llbracket 1, \mu-1 \rrbracket$, such that

$$
\left|U_{1}^{(k+1)}(t)\right| \leq a_{\mu} \tilde{\Upsilon}_{\mu}+\sum_{i=1}^{\mu-1} a_{i} \tilde{\Upsilon}_{i}\left(a_{\mu}, \ldots, a_{i+1}\right), \quad \forall t \geq 0
$$

Let $\tilde{q}(s):=s^{-(p+1)}$, for all $s>0$. Define $h(t):=1+\|z(t)\|^{2}$, for all $t \geq 0$. With the same notation given in the proof of Proposition 2, one can write $U_{1}(\cdot)$ as

$$
U_{1}(t)=-\sum_{i=1}^{\mu} U_{1 i}(t), \quad \forall t \geq 0,
$$

where, for every $i \in \llbracket 1, \mu \rrbracket$,

$$
U_{1 i}(t):=\quad Q_{i, \mu} b_{0, i}^{T} y_{i}(t)\left[g \circ f_{i}\right](t)[\tilde{q} \circ h](t), \quad \forall t \geq 0 .
$$

As in the proof of Proposition 2, we next show that for each $i \in \llbracket 1, \mu \rrbracket$, there exist continuous functions $c_{i, l}: \mathbb{R}_{>0}^{\mu-l} \rightarrow \mathbb{R}_{>0}, l \in \llbracket 1, i \rrbracket$, such that, for all $t \geq 0$,

$$
\left|U_{1 i}^{(k+1)}(t)\right| \leq \sum_{l=1}^{i} a_{l} c_{i, l}\left(a_{\mu}, \ldots, a_{l+1}\right),
$$


$c_{i, \mu}$ is actually a constant independent of $a_{\mu}$, we write it as $c_{i, \mu}\left(a_{\mu}, a_{\mu+1}\right)$ for the sake of notation homogeneity. For $i \in \llbracket 1, \mu \rrbracket$, we apply Leibniz's rule to (54) and obtain that the $(k+1)$-th time derivative of $U_{1 i}(\cdot)$ is given, for all $t \geq 0$, by

$$
U_{1 i}^{(k+1)}(t)=a_{i} Q_{i+1, \mu}\left(\sum_{l_{1}=0}^{k+1} \sum_{l_{2}=0}^{l_{1}}\left(\begin{array}{c}
k+1 \\
l_{1}
\end{array}\right)\left(\begin{array}{c}
l_{1} \\
l_{2}
\end{array}\right)[\tilde{q} \circ h]^{\left(k+1-l_{1}\right)}(t)\left[g \circ f_{i}\right]^{\left(l_{2}\right)}(t) b_{0, i}^{T} y_{i}^{\left(l_{1}-l_{2}\right)}(t)\right) .
$$

Then, to get (55), it is sufficient to show that :

a) there exists $C>0$ such that, for any $\tilde{l} \in \llbracket 0, k+1 \rrbracket$ and for all $t \geq 0$,

$$
\left|[\tilde{q} \circ h]^{(\tilde{l})}(t)\right| \leq C[\tilde{q} \circ h](t) .
$$

b) for each $i \in \llbracket 1, \mu \rrbracket$, there exist $\Psi_{i}, \Theta_{i}, \Phi_{i}: \mathbb{R}_{>0}^{\mu-i} \rightarrow \mathbb{R}_{>0}$, and $v_{i, j}: \mathbb{R}_{>0}^{\mu-j} \rightarrow \mathbb{R}_{>0}$ for $j \in \llbracket 1, i \rrbracket$ such that, for any $\tilde{l} \in \llbracket 0, k+1 \rrbracket$ and for all $t \geq 0$,

$$
\left\|y_{i}^{(\tilde{l})}(t)\right\| \leq \bar{\Psi}_{i}\left(a_{\mu}, \ldots, a_{i+1}\right) \sum_{l=i}^{\mu}\left\|y_{l}(t)\right\|+\Theta_{i}\left(a_{\mu}, \ldots, a_{i+1}\right)\|z(t)\|+\bar{\Phi}_{i}\left(a_{\mu}, \ldots, a_{i+1}\right)+\sum_{l=1}^{i} a_{l} \tilde{v}_{l, i}\left(a_{\mu}, \ldots, a_{l+1}\right) .
$$

c) for each $i \in \llbracket 1, \mu \rrbracket$, there exist $\Gamma_{i}, \theta_{i}: \mathbb{R}_{>0}^{\mu-i} \rightarrow \mathbb{R}_{>0}$, and $\Gamma_{i, j}: \mathbb{R}_{>0}^{\mu-j} \rightarrow \mathbb{R}_{>0}$ for $j \in \llbracket 1, i \rrbracket$ such that, for any $\tilde{l} \in \llbracket 0, k+1 \rrbracket$ and for all $t \geq 0$,

$$
\left|\left[g \circ f_{i}\right]^{(\tilde{l})}(t)\right| \leq\left[g \circ f_{i}\right](t)\left(\Gamma_{i}\left(a_{\mu}, \ldots, a_{i+1}\right)+\sum_{l=1}^{i} a_{l} \tilde{v}_{l, i}\left(a_{\mu}, \ldots, a_{l+1}\right)+\theta_{i}\left(a_{\mu}, \ldots, a_{i+1}\right)\|z(t)\|^{2 \tilde{l}}\right) .
$$

We now establish $a$ ). From an argument of induction using differentiation of $z$-subsystem (48b) coupled with the fact that $\kappa$ is $p$-bounded feedback law, it can easily be shown that there exist $C_{0}, C_{1}>0$ such that for any $\tilde{l} \in \llbracket 1, k+1 \rrbracket$ and for any $t \geq 0$,

$$
\left\|z^{(\tilde{l})}(t)\right\| \leq C_{0}+C_{1}\|z(t)\|
$$

Using the Leibniz rule, it can be establish that there exist $\tilde{C}_{0}, \tilde{C}_{1}>0$ such that, for any $\tilde{l} \in \llbracket 1, k+1 \rrbracket$,

$$
\left|h^{(\tilde{l})}(t)\right| \leq \tilde{C}_{0}+\tilde{C}_{1}\|z(t)\|^{2},
$$

for all $t \geq 0$. Thanks to Faá Di Bruno Formula (Lemma[5] applied to $[q \circ h]$, item $a$ ) follows.

We now deal with item $b$ ). From Lemma 4 and an induction argument using differentiation of system (48a), one can obtain the following statement: for any $l_{1} \in \llbracket 1, k+1 \rrbracket, i \in \llbracket 1, \mu \rrbracket$, there exist continuous functions $\bar{\Psi}_{l_{1}, i, l}: \mathbb{R}_{>0}^{\mu-i} \rightarrow \mathbb{R}_{>0}, l \in \llbracket i+1, \mu \rrbracket, \bar{\Phi}_{l_{1}, i, l}: \mathbb{R}_{>0}^{\mu-i} \rightarrow \mathbb{R}_{>0}, \quad l \in \llbracket 0, p \rrbracket, \bar{\Theta}_{l_{1}, i, l}: \mathbb{R}_{>0}^{\mu-i} \rightarrow \mathbb{R}_{>0}, \quad l \in$ $\llbracket 0, p \rrbracket$, and $\bar{\Xi}_{l_{1}, i, l}: \mathbb{R}_{>0}^{\mu-i} \rightarrow \mathbb{R}_{>0}, \quad l \in \llbracket 0, p \rrbracket$, such that, for all $t \geq 0$,

$$
\begin{aligned}
\left\|y_{i}^{\left(l_{1}\right)}(t)\right\| & \leq \sum_{l=i}^{\mu} \bar{\Psi}_{l_{1}, i, l}\left(a_{\mu}, \ldots, a_{i+1}\right)\left\|y_{l}(t)\right\|+\bar{\Theta}_{l_{1}, i, l}\left(a_{\mu}, \ldots, a_{i+1}\right)\|z(t)\| \\
& +\sum_{l=0}^{l_{1}-1} \bar{\Phi}_{l_{1}, i, l}\left(a_{\mu}, \ldots, a_{i+1}\right)\left|U_{1}^{(l)}(t)\right|+\bar{\Xi}_{l_{1}, i, l}\left(a_{\mu}, \ldots, a_{i+1}\right)\left\|U_{2}^{\left(l_{1}\right)}(t)\right\| .
\end{aligned}
$$

So, using the inductive hypothesis and the fact that $\kappa$ is a $p$-bounded feedback law, one can obtain item $b$ ).

Proceeding as in Proposition 2, one can get item $c$ ). This ends the proof of Proposition 3 . 


\section{Appendix}

\subsection{Proof of Lemma 2}

Let $\varepsilon>1$ and $\beta>0$. We first prove forward completeness of

$$
\dot{x}=-\beta \frac{x}{\left(1+x^{2}\right)^{1 / 2}}+d_{1}
$$

in response to any locally bounded function $d_{1}(\cdot)$. For this, let $V(x):=x^{2} / 2$. Its derivative along trajectories of (56) satisfies

$$
\dot{V}(x)=-\beta \frac{x^{2}}{\left(1+x^{2}\right)^{1 / 2}}+x^{T} d_{1}(t) .
$$

Then, a straightforward computation leads to $\dot{V}(x) \leq V(x)+d_{1}(t)^{2}$ and forward completeness follows using classical comparison results. Moreover when $d_{1}=0$, (57) ensures that the origin of (56) is G.A.S.

We then prove the $\operatorname{SISS}_{L}\left(\beta / 2, \frac{2 \varepsilon}{\beta}\right)$ property of the system (56) with respect to $d_{1}(\cdot)$. Given $\delta \leq \beta / 2$, let $d_{1}$ be a bounded measurable function on $\mathbb{R}_{\geq 0}$ eventually bounded by $\delta$. Since the system is forward complete, we can consider without loss of generality that $d_{1}(t) \leq \delta$ for all $t \geq 0$. From (57) and the fact that $\left(1+x^{2}\right)^{1 / 2} \leq 1+|x|$, one can obtain that

$$
\dot{V}(x)=-\beta \frac{x^{2}}{\left(1+x^{2}\right)^{1 / 2}}+\frac{1}{\left(1+x^{2}\right)^{1 / 2}}\left(\left|d_{1}(t)\right||x|+\left|d_{1}(t)\right| x^{2}\right) .
$$

Observing that

$$
\frac{\left|d_{1}(t)\right| x^{2}}{\left(1+x^{2}\right)^{1 / 2}} \leq \frac{\beta x^{2}}{2\left(1+x^{2}\right)^{1 / 2}}
$$

it follows that

$$
\dot{V}(x) \leq-\beta \frac{|x|}{\left(1+x^{2}\right)^{1 / 2}}\left(|x|-\frac{2}{\beta} \delta\right) .
$$

Consequently, $\dot{V}<0$ whenever $|x|>\frac{2 \delta}{\beta}$. It follows that every trajectory of (10) eventually enters and remains in the set $S=\left\{x \in \mathbb{R}: x^{2} \leq \varepsilon^{2}\left(\frac{2 \delta}{\beta}\right)^{2}\right\}$ (indeed, $\dot{V}<0$ for all $x \notin S$ and $x \in \partial S$ ). Thus Lemma 2 can be easily established.

\subsection{Proof of Lemma 3}

Let $\omega>0$. Given any $0<\beta<1$, let $A_{\beta}:=\omega A_{0}-\beta b_{0} b_{0}^{T}$, which is Hurwitz since $A_{0}$ is skew-symmetric and $\left(A_{0}, b_{0}\right)$ is controllable. Therefore there exists a symmetric positive definite matrix $P_{\beta}$ satisfying the following Lyapunov equation

$$
P_{\beta} A_{\beta}+A_{\beta}^{T} P_{\beta}=-\mathbb{I}_{2} .
$$

A simple computation gives

$$
P_{\beta}=\left(\begin{array}{cc}
\frac{\beta}{2 \omega^{2}}+\frac{1}{\beta} & \frac{1}{2 \omega} \\
\frac{1}{2 \omega} & \frac{1}{\beta}
\end{array}\right) .
$$


The smallest and largest eigenvalues of $P_{\beta}$ denoted by $\underline{\sigma}_{\beta}$ and $\bar{\sigma}_{\beta}$ respectively are given by

$$
\begin{aligned}
& \underline{\sigma}_{\beta}:=\beta\left\|P_{\beta} b_{0}\right\|^{2}-\frac{\beta}{2 \omega}\left\|P_{\beta} b_{0}\right\|, \\
& \bar{\sigma}_{\beta}:=\beta\left\|P_{\beta} b_{0}\right\|^{2}+\frac{\beta}{2 \omega}\left\|P_{\beta} b_{0}\right\|,
\end{aligned}
$$

with

$$
\left\|P_{\beta} b_{0}\right\|=\sqrt{\frac{1}{4 \omega^{2}}+\frac{1}{\beta^{2}}} .
$$

Define $V: \mathbb{R}^{2} \rightarrow \mathbb{R}_{\geq 0}$ as

$$
V(x):=x^{T} P_{\beta} x+\frac{\left(\bar{\sigma}_{\beta}+\underline{\sigma}_{\beta}\right)}{3}\left(\left(1+\|x\|^{2}\right)^{3 / 2}-1\right), \quad \forall x \in \mathbb{R}^{2} .
$$

Given $C>1$, let $\alpha_{1}$ and $\alpha_{2}$ be class $\mathscr{K}_{\infty}$ functions given by

$$
\begin{aligned}
& \alpha_{1}(r):=\frac{\left(\bar{\sigma}_{\beta}+\underline{\sigma}_{\beta}\right)}{C} \max \left\{r^{2}, r^{3}\right\}, \\
& \alpha_{2}(r):=C\left(\bar{\sigma}_{\beta}+\underline{\sigma}_{\beta}\right) \max \left\{r^{2}, r^{3}\right\} .
\end{aligned}
$$

There exists $C>1$ such that

$$
\alpha_{1}(\|x\|) \leq V(x) \leq \alpha_{2}(\|x\|), \quad \forall x \in \mathbb{R}^{2} .
$$

Moreover, there exists a constant $M>0$, independent of $\beta$, such that

$$
\alpha_{1}^{-1} \circ \alpha_{2}(r) \leq M r, \quad \forall r \geq 0 .
$$

Proceeding as in the proof of Lemma2, forward completeness of

$$
\dot{x}=\omega A_{0} x-\beta b_{0} \frac{b_{0}^{T} x}{\left(1+\|x\|^{2}\right)^{1 / 2}}+d_{1}
$$

can easily be derived in response to any locally measurable bounded function $d_{1}$. We next show that the system (63) is $\operatorname{SISS}_{L}(\beta \Gamma, N / \beta)$ with respect to $d_{1}$, for some $N>0$ and with

$$
\Gamma:=\frac{1}{8\left(\frac{1}{4 \omega^{2}}+1\right)}
$$

Since (63) is forward complete, we can assume without loss of generality that $d_{1}$ satisfies $\left\|d_{1}(t)\right\| \leq \delta, \quad \forall t \geq$ 0 , for some $\delta \leq \beta \Gamma$. Consider the Lyapunov function $V: \mathbb{R}^{2} \rightarrow \mathbb{R}$ defined in (61). By noticing that (63) can be rewritten as

$$
\dot{x}=A_{\beta} x+\beta b_{0} b_{0}^{T} x\left(1-\frac{1}{\left(1+\|x\|^{2}\right)^{1 / 2}}\right)+d_{1},
$$


one gets that the time derivative of $V$ along trajectories of (63) satisfies

$$
\begin{aligned}
\dot{V}= & x^{T} P_{\beta}\left(A_{\beta} x+\beta b_{0} b_{0}^{T} x\left(1-\frac{1}{\left(1+\|x\|^{2}\right)^{1 / 2}}\right)+d_{1}\right)+\left(x^{T} A_{\beta}^{T}+\beta b_{0}^{T} b_{0}^{T} x\left(1-\frac{1}{\left(1+\|x\|^{2}\right)^{1 / 2}}\right)+d_{1}^{T}\right) P_{\beta} x \\
& +\left(\bar{\sigma}_{\beta}+\underline{\sigma}_{\beta}\right)\left(1+\|x\|^{2}\right)^{1 / 2}\left(-\beta \frac{\left(b_{0}^{T} x\right)^{2}}{\left(1+\|x\|^{2}\right)^{1 / 2}}+x^{T} d_{1}\right) .
\end{aligned}
$$

Since $P_{\beta}$ is a symmetric matrix satisfying the Lyapunov equation $[60$, it follows that $\dot{V}=-\|x\|^{2}+2 \beta x^{T} P_{\beta} b_{0} b_{0}^{T} x\left(1-\frac{1}{\left(1+\|x\|^{2}\right)^{1 / 2}}\right)+2 x^{T} P_{\beta} d_{1}-\beta\left(\bar{\sigma}_{\beta}+\underline{\sigma}_{\beta}\right)\left(b_{0}^{T} x\right)^{2}+\left(\bar{\sigma}_{\beta}+\underline{\sigma}_{\beta}\right)\left(1+\|x\|^{2}\right)^{1 / 2} x^{T} d_{1}$.

By completing the squares it holds that, for all $t \geq 0$,

$$
\left|2 \beta x^{T} P_{\beta} b_{0} b_{0}^{T} x\left(1-\frac{1}{\left(1+\|x\|^{2}\right)^{1 / 2}}\right)\right| \leq \frac{\|x\|^{2}}{2}+2 \beta^{2}\left\|P_{\beta} b_{0}\right\|^{2}\left(b_{0}^{T} x\right)^{2} .
$$

Therefore, one can get that

$$
\dot{V} \leq-\frac{1}{2}\|x\|^{2}+2 x^{T} P d_{1}+2 \beta\left\|P b_{0}\right\|^{2}\left(1+\|x\|^{2}\right)^{1 / 2} x^{T} d_{1} .
$$

Using the fact that $\left(1+\|x\|^{2}\right)^{1 / 2} \leq 1+\|x\|$ for all $x \in \mathbb{R}^{2}$, and exploiting (64), it follows that

$$
\dot{V} \leq-\frac{1}{4}\|x\|^{2}+2\|x\| \delta\left(2 \beta\left\|P_{\beta} b_{0}\right\|^{2}+\frac{\beta}{2 \omega}\left\|P_{\beta} b_{0}\right\|\right)
$$

Consequently, it holds that $\dot{V}<0$ whenever $\|x\|>8 \delta\left(2 \beta\left\|P_{\beta} b_{0}\right\|^{2}+\frac{\beta}{2 \omega}\left\|P_{\beta} b_{0}\right\|\right)$. Let $\mu>1$ and set $r:=$ $8 \mu\left(2 \beta\left\|P_{\beta} b_{0}\right\|^{2}+\frac{\beta}{2 \omega}\left\|P_{\beta} b_{0}\right\|\right)$. Define $S:=\left\{x \in \mathbb{R}^{2}: V(x) \leq \alpha_{2}(r \delta)\right\}$. If $x \notin S$ then $\|x\|>r \delta$. Consequently, any trajectory eventually enters and stay in $S$. Moreover, we have that $\alpha_{1}(\|x(t)\|) \leq_{e v} V(x(t)) \leq \alpha_{2}(r \delta)$. From (62), it follows that $\|x(t)\| \leq_{e v} r M \delta$. Moreover, one can see that there exists a constant $D>0$ such that for any $\beta \leq 1$ we have $r \leq \frac{D}{\beta}$. So we obtain

$$
\|x(t)\| \leq_{e v} \frac{N \delta}{\beta}
$$

for some $N>0$, which concludes the proof.

\subsection{Faà Di Bruno's Formula}

Lemma 5 (Faà Di Bruno's formula, [21], p. 96). For $k \in \mathbb{N}$, let $\phi \in C^{k}\left(\mathbb{R}_{\geq 0}, \mathbb{R}\right)$ and $\rho \in C^{k}(\mathbb{R}, \mathbb{R})$. Then the $k$-th order derivative of the composite function $\rho \circ \phi$ is given by

$$
[\rho \circ \phi]^{(k)}(t)=\sum_{a=1}^{k} \rho^{(a)}(\phi(t)) B_{k, a}\left(\phi^{(1)}(t), \ldots, \phi^{(k-a+1)}(t)\right)
$$


where $B_{k, a}$ is the Bell polynomial given by

$$
B_{k, a}\left(\phi^{(1)}(t), \ldots, \phi^{(k-a+1)}(t)\right):=\sum_{\delta \in \mathscr{P}_{k, a}} c_{\delta} \prod_{l=1}^{k-a+1}\left(\phi^{(l)}(t)\right)^{\delta_{l}},
$$

where $\mathscr{P}_{k, a}$ denotes the set of $(k-a+1)-$ tuples $\delta:=\left(\delta_{1}, \delta_{2}, \ldots, \delta_{k-a+1}\right)$ of positive integers satisfying

$$
\begin{aligned}
& \delta_{1}+\delta_{2}+\ldots+\delta_{k-a+1}=a, \\
& \delta_{1}+2 \delta_{2}+\ldots+(k-a+1) \delta_{k-a+1}=k, \\
& c_{\delta}:=\frac{k !}{\left(\delta_{1} ! \cdots \delta_{k-a+1} !(1 !)^{\delta_{1}} \cdots((k-a+1) !)^{\delta_{k-a+1}}\right)} .
\end{aligned}
$$

\section{References}

[1] A. Saberi, A. Stoorvogel, and P. Sannuti, Internal and External Stabilization of Linear Systems with Constraints, ser. Systems \& Control: Foundations \& Applications. Birkhäuser Boston, 2012.

[2] A. T. Fuller, "In-the-large stability of relay and saturating control systems with linear controllers," Int. J. of Control, vol. 10, no. 4, pp. 457-480, 1969.

[3] H. Sussmann and Y. Yang, "On the stabilizability of multiple integrators by means of bounded feedback controls," in IEEE Conf. Decision Contr., vol. 1, Dec 1991.

[4] E. P. Ryan, Optimal relay and saturating control system synthesis / E.P. Ryan. P. Peregrinus on behalf of the Institution of Electrical Engineers Stevenage, UK ; New York, 1982.

[5] A. R. Teel, "Global stabilization and restricted tracking for multiple integrators with bounded controls," Syst. Contr. Letters, vol. 18, no. 3, pp. 165 - 171, 1992.

[6] H. J. Sussmann, E. D. Sontag, and Y. Yang, "A general result on the stabilization of linear systems using bounded controls," IEEE Trans. Autom. Control, vol. 39, pp. 2411-2425, 1994.

[7] Y. Lin and E. D. Sontag, "Control-Lyapunov universal formulas for restricted inputs," Control-Theory and Advanced Technology, vol. 10, pp. 1981-2004, 1995.

[8] A. Saberi, Z. Lin, and A. Teel, "Control of linear systems with saturating actuators," IEEE Trans. Autom. Control, vol. 41, no. 3, pp. 368-378, 2002.

[9] A. Saberi, P. Hou, and A. A. Stoorvogel, "On simultaneous global external and global internal stabilization of critically unstable linear systems with saturating actuators," IEEE Trans. Autom. Control, vol. 45, no. 6, pp. 1042-1052, 2000.

[10] A. Megretski, "BIBO output feedback stabilization with saturated control," in IFAC World Congress, 1996, pp. 435-440.

[11] Y. Chitour, M. Harmouche, and S. Laghrouche, " $L_{p}$-Stabilization of Integrator Chains Subject to Input Saturation Using Lyapunov-Based Homogeneous Design," SIAM Journal on Control and Optimization, vol. 53, no. 4, pp. 2406-2423, 2015. 
[12] R. Azouit, A. Chaillet, Y. Chitour, and L. Greco, "Strong iISS for a class of systems under saturated feedback," To appear in Automatica, 2016.

[13] R. Freeman and L. Praly, "Integrator backstepping for bounded controls and control rates," IEEE Trans. Autom. Control, vol. 43, no. 2, pp. 258-262, 1998.

[14] J. M. Shewchun and E. Feron, "High performance control with position and rate limited actuators," International Journal of Robust and Nonlinear Control, vol. 9, no. 10, pp. 617-630, 1999.

[15] J. Solís-Daun, R. Suárez, and J. Álvarez-Ramírez, "Global stabilization of nonlinear systems with inputs subject to magnitude and rate bounds: A parametric optimization approach," SIAM Journal on Control and Optimization, vol. 39, no. 3, pp. 682-706, 2000.

[16] T. Lauvdal, R. Murray, and T. Fossen, "Stabilization of integrator chains in the presence of magnitude and rate saturations: a gain scheduling approach," in IEEE Conf. Decision Contr., vol. 4, Dec 1997, pp. 4004-4005 vol.4.

[17] Z. Lin, "Semi-global stabilization of linear systems with position and rate-limited actuators," Systems \& Control Letters, vol. 30, no. 1, pp. 1-11, 1997.

[18] J. Gomes da Silva, J.M., S. Tarbouriech, and G. Garcia, "Local stabilization of linear systems under amplitude and rate saturating actuators," IEEE Trans. Autom. Control, vol. 48, no. 5, pp. 842-847, May 2003.

[19] A. R. Teel, "A nonlinear small gain theorem for the analysis of control systems with saturation," IEEE Trans. Autom. Control, vol. 41, no. 9, pp. 1256-1270, 1996.

[20] J. Laporte, A. Chaillet, and Y. Chitour, "Global stabilization of classes of linear control systems with bounds on the feedback and its successive derivatives," Submitted to Systems and Control Letters, 2015.

[21] M. Hazewinkel, Encyclopaedia of Mathematics (1), ser. Encyclopaedia of Mathematics: An Updated and Annotated Translation of the Soviet "Mathematical Encyclopaedia". Springer, 1987. 